\title{
Fokker-Planck analysis of transverse collective instabilities in electron storage rings
}

\author{
Ryan R. Lindberg \\ Advanced Photon Source, Argonne National Laboratory, Argonne, Illinois 60439, USA
}

(Received 10 June 2016; published 30 December 2016)

\begin{abstract}
We analyze single bunch transverse instabilities due to wakefields using a Fokker-Planck model. We first expand on the work of T. Suzuki, Part. Accel. 12, 237 (1982) to derive the theoretical model including chromaticity, both dipolar and quadrupolar transverse wakefields, and the effects of damping and diffusion due to the synchrotron radiation. We reduce the problem to a linear matrix equation, whose eigenvalues and eigenvectors determine the collective stability of the beam. We then show that various predictions of the theory agree quite well with results from particle tracking simulations, including the threshold current for transverse instability and the profile of the unstable mode. In particular, we find that predicting collective stability for high energy electron beams at moderate to large values of chromaticity requires the full FokkerPlanck analysis to properly account for the effects of damping and diffusion due to synchrotron radiation.
\end{abstract}

DOI: 10.1103/PhysRevAccelBeams.19.124402

\section{INTRODUCTION}

Understanding, predicting, and controlling collective instabilities is an important part of storage ring design and operation. Single bunch transverse instabilities are of particular importance in high-energy electron storage rings, as they typically set the limit on the maximum achievable current [1-4]. The standard analysis of these instabilities decomposes the linearized Vlasov equation into normal modes, and then stability is determined by comparing the maximum growth rate with the transverse synchrotron and Landau damping rates (see, e.g., [5-9]). However, synchrotron emission results in both damping and diffusion in phase space, so that when synchrotron radiation provides the dominant damping mechanism it can render the Vlasov model incomplete. This is often the case for high energy electron storage rings, in which case a Fokker-Planck description must be employed to accurately predict stability. This paper builds on the work of Ref. [10] to develop a more complete Fokker-Planck analysis of transverse stability, where particular attention is paid to the dynamics at large chromaticity.

This paper is organized as follows. We derive the linear equation of transverse stability from the Fokker-Planck equation in Sec. II. We work out the derivation in some detail, even though most of the elements of our calculation can be found in the papers $[5,6,10-12]$ and the books $[7,8]$. The (to our knowledge) new parts of the analysis include the explicit treatment of the chromaticity via a canonical

Published by the American Physical Society under the terms of the Creative Commons Attribution 4.0 International license. Further distribution of this work must maintain attribution to the author(s) and the published article's title, journal citation, and DOI. transformation that naturally introduces the head-tail phase in Sec. II A, and the inclusion of the quadrupolar wakefield term throughout (although some of the latter was hinted at in the Ph.D. theses $[13,14])$. In Sec. II B we include the Fokker-Planck dynamics, which leads to the equilibrium and perturbed distribution discussed in Sec. II C. Those who are familiar with the linearization of the Vlasov equation can probably skim Sec. II A, while those who have experience with the Fokker-Planck analysis can probably skip directly to the linearized equation of transverse stability derived in Sec II D and given by Eq. (51).

In Sec. III we apply Sacherer's formalism [5,6] to derive the matrix equation that governs transverse stability to wakefields in an electron storage ring, which we write in Eq. (72). This procedure is quite similar to that first applied to the Fokker-Planck equation in Ref. [10], but we more fully investigate arbitrary values of chromaticity. We reproduce Ref. [10]'s result showing that the damping of high-order modes increases linearly with mode number, but also derive a much simpler expression for the diffusioninduced coupling between the modes, and find that these coupling terms do not play a significant role in the dynamics.

Section IV compares predictions from our analysis to those of tracking simulations using the code ELEGANT [15]. For this comparison we use the multibend achromat (MBA) upgrade lattice planned for the Advanced Photon Source as our model storage ring [16], and the anticipated resistive wall transverse impedance as our source of collective instabilities; application to other lattices and/or impedances is relatively straightforward. We begin by reviewing the small-chromaticity limit assuming a purely dipolar impedance, where we find very similar results to those of the standard analysis of the transverse mode coupling instability [17-20] and that of Ref. [10]. 
Next, we investigate increasing the current limit further by operating at relatively high chromaticity in Sec. IV B. The large, positive chromaticity stabilizes the low-order modes, so that higher-order modes determine the dynamics. Because higher-order modes are more susceptible to damping and diffusion, we find that in the high-chromaticity limit including the full Fokker-Planck operator is essential for properly predicting the stability within Sacherer's matrix formulation. Indeed, our theoretical predictions closely match simulation results over a wide range of chromaticity, both in terms of the current at which the beam goes unstable, and in predicting the phase space distribution of the unstable beam. In addition, we make a brief comparison of our predictions with those of the "post head-tail" theory [21].

Finally, we extend the simulations to first include the quadrupolar transverse impedance due to the nearly flat, small gap insertion device chambers in Sec. IV C, and then the longitudinal potential provided by the longitudinal wakefield in Sec. IV D. The former quadrupolar contribution changes the frequency shifts of the modes as explained in Ref. [22]; both our theory and simulation predict that including $Z_{Q}$ can increase the maximum stable current by $10 \%$ to $40 \%$. We also show that the longitudinal impedance can be included with a straightforward extension of the theory, but only if it is a small perturbation to the linear dynamics.

We believe that our simulations show that the linearized Fokker-Planck theory describes transverse, single-bunch instabilities in electron storage rings provided one knows both the transverse impedance and the equilibrium longitudinal distribution. The present work assumes that the longitudinal potential is dominated by the linear rf focusing, so that it directly applies only to rings in which the longitudinal impedance is a small perturbation, and to $\mathrm{rf}$ systems that do not employ bunch-lengthening harmonic rf cavities. It appears that the present theory can be extended to include the latter harmonic rf with only a modest increase in computational complexity, but generalizing it to encompass an arbitrary longitudinal impedance may not be reasonable. Finally, many next-generation, low-emittance storage rings have large second order chromatic effects that we plan to address in a subsequent publication.

\section{GOVERNING EQUATIONS}

Under Hamiltonian (conservative) flow, the evolution of the electron distribution function $F$ is given by

$$
\frac{\partial F}{\partial s}+\{F, \mathcal{H}\}=0
$$

where $s$ is the propagation coordinate along the ring and the canonical Poisson bracket is defined in terms of the positions $\boldsymbol{X}$ and momenta $\boldsymbol{P}$ via

$$
\{F, \mathcal{H}\} \equiv \frac{\partial \mathcal{H}}{\partial \boldsymbol{P}} \cdot \frac{\partial F}{\partial \boldsymbol{X}}-\frac{\partial \mathcal{H}}{\partial \boldsymbol{X}} \cdot \frac{\partial F}{\partial \boldsymbol{P}}
$$

Our convention is to normalize $F$ such that integrating over all phase-space coordinates $\mathcal{Z}=(\boldsymbol{X}, \boldsymbol{P})$ is unity.

In electron storage rings, however, the Hamiltonian dynamics is modified by the emission of synchrotron radiation, which leads to a damping and diffusion in phase space. As shown in, e.g., [23], and derived using an alternate method in Appendix A, this damping and diffusion can be included by modifying (1) to

$$
\frac{\partial F}{\partial s}+\{F, \mathcal{H}\}=\left.\frac{\partial F}{\partial s}\right|_{\text {F.P. }},
$$

where the right-hand side represents a Fokker-Planck operator that gives the damping and diffusion associated with synchrotron emission.

We assume that the transverse degrees of freedom are nearly uncoupled, in which case we need only consider two degrees of freedom and take $X=(x, z)$, where $x$ is the horizontal transverse coordinate and the longitudinal comoving coordinate $z \equiv s-c t$. Their respective momenta are the transverse angle $x^{\prime}$ and the negative energy deviation $p_{z} \equiv-\left(\gamma-\gamma_{0}\right) / \gamma_{0}$, where $\gamma_{0}$ is the reference Lorentz factor; we choose the negative energy deviation rather than the usual $\delta \equiv\left(\gamma-\gamma_{0}\right) / \gamma_{0}$ so that the associated Hamiltonian is positive for particles above transition [in this case particles with positive $p_{z}$ (lower energy) travel a shorter path and are displaced a positive amount in $z$ after one turn].

We consider Hamiltonian motion that encompasses the linear optics of the lattice, the linearized rf acceleration, the dependence of the transverse motion on the energy due to chromaticity, and the forces due to wakefields. Furthermore, we assume that the chromatic and wakefield potentials constitute a perturbation over one turn, in which case we can approximate $\mathcal{H}$ by its value integrated over one turn. Hence, we write

$$
\mathcal{H}=\frac{1}{\mathcal{C}_{R}} \oint d s \mathcal{H}\left(x_{\beta}, z, x_{\beta}^{\prime}, p_{z} ; s\right),
$$

where $\mathcal{H}$ is the single particle Hamiltonian and $\mathcal{C}_{R}$ is the ring circumference; in betatron coordinates $x_{\beta} \equiv x+\eta_{x} p_{z}$ and $x_{\beta}^{\prime} \equiv x^{\prime}+\eta_{x}^{\prime} p_{z}$, where $\eta_{x}(s)$ and $\eta_{x}^{\prime}(s)$ are the dispersion function and its derivative, respectively, we have

$$
\begin{aligned}
\mathcal{H}= & \frac{1}{2} x_{\beta}^{\prime 2}+\frac{1}{2} K(s) x_{\beta}^{2}+\frac{\alpha_{c}}{2} p_{z}^{2}+\mathcal{V}_{z}(z ; s) \\
& +\mathcal{V}_{\text {chrom }}\left(p_{z}, x_{\beta}, ; s\right)+\mathcal{V}_{\text {wake }}\left(z, x_{\beta} ; s\right)
\end{aligned}
$$

Here, $K(s)$ is the linear focusing due to quadrupoles, $\alpha_{c}$ is the momentum compaction (we assume that we are well above transition, $\left.\alpha_{c} \gg 1 / \gamma^{2}\right)$, and $\mathcal{V}_{z}, \mathcal{V}_{\text {chrom }}$, and $\mathcal{V}_{\text {wake }}$ are 
the conservative synchrotron, chromatic, and transverse wakefield potentials, respectively.

Finally, dynamical consistency demands that we also integrate the Fokker-Planck diffusion and damping over one turn. In the next two sections we first detail the Hamiltonian dynamics, and then determine the FokkerPlanck operator $\partial F /\left.\partial s\right|_{\text {F.P. }}$.

\section{A. Hamiltonian dynamics}

We will find it convenient to analyze the transverse stability by first transforming to the transverse action-angle coordinates, $\left(x_{\beta}, x_{\beta}^{\prime}\right) \rightarrow(\Psi, \mathcal{J})$. These are given in terms of the Courant-Snyder beam envelope function $\beta_{z}(s)$ [24] via

$$
\begin{gathered}
x_{\beta}=\sqrt{2 \beta_{x} \mathcal{J}} \cos \Psi \\
x_{\beta}^{\prime}=-\sqrt{\frac{2 \mathcal{J}}{\beta_{x}}} \sin \Psi+\frac{\beta_{x}^{\prime}}{2} \sqrt{\frac{2 \mathcal{J}}{\beta_{x}}} \cos \Psi,
\end{gathered}
$$

while the new Hamiltonian becomes

$$
\mathcal{H}=\frac{1}{\beta_{x}} \mathcal{J}+\frac{\alpha_{c}}{2} p_{z}^{2}+\mathcal{V}_{z}+\mathcal{V}_{\text {chrom }}+\mathcal{V}_{\text {wake }}
$$

Now, we write out the averaged Hamiltonian. First, the uncoupled part of $\mathcal{H}$ is

$$
\begin{aligned}
& \frac{1}{\mathcal{C}_{R}} \oint d s\left[\frac{\mathcal{J}}{\beta_{x}}+\frac{\alpha_{c}}{2} p_{z}^{2}+\mathcal{V}_{z}(z ; s)\right]=\frac{\omega_{\beta}}{c} \mathcal{J}+\frac{\alpha_{c}}{2} p_{z}^{2}+V_{z}(z) \\
& \quad \equiv \frac{\omega_{\beta}}{c} \mathcal{J}+\mathcal{H}_{z}\left(z, p_{z}\right)
\end{aligned}
$$

where $\omega_{\beta}$ is the betatron frequency and we have defined the longitudinal Hamiltonian $\mathcal{H}_{z}$ as shown. Second, we consider the chromatic potential $\mathcal{V}_{\text {chrom, }}$, which comes from chromatic aberrations in the focusing quadrupoles and in the sextupoles magnets designed to correct these aberrations. For a sextupole with magnetic field components $B_{x}=(m c / e) 2 b_{2} x y$ and $B_{y}=(m c / e) 2 b_{2}\left(x^{2}-y^{2}\right)$, the chromatic potential is [25]

$$
\begin{aligned}
V_{\text {chrom }} & =\oint d s\left[K(s)-2 b_{2}(s) \eta_{x}(s)\right] \frac{x_{\beta}^{2} p_{z}}{2} \\
& =\mathcal{J}[1+\cos (2 \Psi)] p_{z} \frac{1}{\mathcal{C}_{R}} \oint d s \frac{\beta_{x}}{2}\left[K-2 b_{2} \eta_{x}\right] \\
& =-\frac{\omega_{0} \xi_{x}}{c} \mathcal{J}[1+\cos (2 \Psi)] p_{z},
\end{aligned}
$$

where we have introduced the revolution frequency $\omega_{0} \equiv$ $2 \pi c / \mathcal{C}_{R}$ and the chromaticity

$$
\xi_{x} \equiv-\frac{1}{4 \pi} \oint d s \beta_{x}(s)\left[K(s)-2 b_{2}(s) \eta_{x}(s)\right]
$$

Third, the wakefield potential is found as follows. We model $\mathcal{V}_{\text {wake }}$ as a short-range (single bunch) force that is comprised of a sum over many localized "wakefield elements." At each wakefield element electrons generate electromagnetic fields that provide a kick to all trailing particles. The total kick due to the $\ell$ th element is obtained by summing over all the source electrons using the electron distribution function $F$, so that the wake potential

$$
\begin{aligned}
\mathcal{V}_{\text {wake }}= & \sum_{\ell} \delta\left(s-s_{\ell}\right) \frac{e^{2} N_{e}}{\gamma m c^{2}} \int d \hat{\mathcal{Z}} F(\hat{\mathcal{Z}} ; s) \\
& \times\left[x_{\beta} \hat{x}_{\beta} W_{D}^{\ell}(z-\hat{z})+\frac{1}{2} x_{\beta}^{2} W_{Q}^{\ell}(z-\hat{z})\right],
\end{aligned}
$$

where $N_{e}$ is the number of electrons in a bunch and we recall that $\mathcal{Z}$ denotes the set of all phase space coordinates. In addition, Eq. (12) divides the transverse wakefield into its dipolar and quadrupolar components $W_{D}$ and $W_{Q}$ in the usual way. For the former $W_{D}$ the kick strength scales linearly with the displacement of the source electron, while for the latter $W_{Q}$ a test electron receives a kick that is proportional to its own $x$ coordinate; this is the general, linearized wakefield produced by vacuum chambers that have left-right and top-bottom mirror symmetry (see, e.g., [26]). Note that both wakefields depend on the distance between the source and test electron, and that in the ultrarelativistic limit the wakefield of any particle can only affect those electrons that are behind it, $W(z-\hat{z})=0$ if $\hat{z}<z$.

Averaging $\mathcal{V}_{\text {wake }}$ over the ring is trivial: we merely get a sum over the various wakefield elements at their location. The interesting part comes when we make the coordinate change to action-angle variables. Using the fact that the Jacobian of a canonical transformation is unity, we find that the transverse wakefield potential (12) becomes

$$
\begin{aligned}
V_{\text {wake }}= & \frac{e^{2} N_{e}}{\gamma m c^{2}} \frac{\omega_{0}}{2 \pi c} \sum_{\ell} \int d \hat{\mathcal{Z}} F(\hat{\mathcal{Z}} ; s) \\
& \times\left\{2 \sqrt{\mathcal{J}} \cos \Psi \sqrt{\hat{\mathcal{J}}} \cos \hat{\Psi} \beta_{x}^{\ell} W_{D}^{\ell}(z-\hat{z})\right. \\
& \left.+\frac{\mathcal{J}}{2}[1+\cos (2 \Psi)] \beta_{x}^{\ell} W_{Q}^{\ell}(z-\hat{z})\right\},
\end{aligned}
$$

and we derive the fact that the effect of the transverse wakefield is scaled by the local beta function. While this $\beta$-function scaling has been well-known for decades, it apparently was first proven in [13], which in fact generalized our Eq. (13) to elements with finite extent in $s$; the expression in Ref. [13] reduces to (13) if the wakefield source has a length much less then $c / \omega_{\beta}$, or alternatively if 
it is nearly constant over that length as might be the case for the resistive wall wake.

Our ultimate interest will be to determine the transverse stability, so that we will concern ourselves with both the equilibrium distribution and with the transverse dynamics associated with the betatron motion $\sim e^{i \Psi}$. In this case the terms $\propto \cos (2 \Psi)$ result in oscillatory corrections that we can, to lowest order, ignore. Hence, we will drop these terms from (10) and (13), and write our ring-averaged Hamiltonian as

$$
\mathcal{H}=\frac{\omega_{\beta}}{c} \mathcal{J}+\frac{\alpha_{c}}{2} p_{z}^{2}+V_{z}-\frac{\omega_{0} \xi_{x}}{c} \mathcal{J} p_{z}+V_{\text {wake }} .
$$

Our next step will be to eliminate the chromatic potential from $\mathcal{H}$ using an appropriate coordinate transformation. We do this using the "type-three" [27,28] mixed-variable generating function given by

$$
S_{3}^{\xi}\left(\bar{\Psi}, \bar{z}, \mathcal{J}, p_{z}\right)=-\bar{\Psi} \mathcal{J}-\bar{z} p_{z}+\frac{\omega_{0} \xi_{x}}{\alpha_{c} c} \bar{z} \mathcal{J},
$$

where bars indicate the new coordinates. The new longitudinal variables are given by

$$
z=-\frac{\partial S_{3}^{\xi}}{\partial p_{z}}=\bar{z} \quad \bar{p}_{z}=-\frac{\partial S_{3}^{\xi}}{\partial \bar{z}}=p_{z}-\frac{\omega_{0} \xi_{x}}{\alpha_{c} c} \mathcal{J}
$$

We will find momentarily that $\overline{\mathcal{J}}=\mathcal{J}$, so that inserting the new longitudinal momentum into (14) cancels the chromatic term $\left(\omega_{0} \xi_{x} / c\right) p_{z} \mathcal{J}$. In addition, the transverse Hamiltonian gets a nonlinear contribution such that

$$
\frac{\omega_{\beta}}{c} \mathcal{J} \rightarrow \frac{\omega_{\beta}}{c} \overline{\mathcal{J}}\left(1-\frac{\omega_{0}^{2} \xi_{x}^{2}}{2 \omega_{\beta} \alpha_{c} c} \overline{\mathcal{J}}\right) .
$$

However, the correction in parentheses is typically entirely negligible (for the APS and APSU it is $\lesssim 10^{-7}$ ), and we neglect the term $\sim \mathcal{J}^{2}$ in what follows.

Along with the new longitudinal coordinates (16), the generating function $S_{3}^{\xi}$ also leads to the transverse coordinates

$$
\Psi=-\frac{\partial S_{3}^{\xi}}{\partial \bar{J}}=\bar{\Psi}-\frac{\omega_{0} \xi_{x}}{\alpha_{c} c} \bar{z} \quad \overline{\mathcal{J}}=-\frac{\partial S_{3}^{\xi}}{\partial \bar{\Psi}}=\mathcal{J} .
$$

We find that the new betatron angle is related to the old one by adding on the so-called "head-tail phase" [29,30]

$$
\frac{\omega_{0} \xi_{x}}{\alpha_{c} c} \bar{z}=\frac{2 \pi \xi_{x}}{\alpha_{c} \mathcal{C}_{R}} z \equiv k_{\xi} z
$$

The head-tail phase arises because the betatron frequency depends linearly on the energy for $\xi_{x} \neq 0$. This chromatic effect leads to the betatron phase accumulating a shift that is proportional to the longitudinal coordinate $z$ as it performs synchrotron oscillations.

In summary, at lowest order the chromatic (head-tail) coordinate transformation eliminates the chromatic part of $\mathcal{H}$ while replacing the betatron phase via $\Psi \rightarrow \bar{\Psi}-k_{\xi} \bar{z}$. The other new coordinates are essentially the same as the old ones, so for simplicity we will only retain the bar on the new betatron phase; the new Hamiltonian is then

$$
\mathcal{H}=\frac{\omega_{\beta}}{c} \mathcal{J}+\mathcal{H}_{z}\left(z, p_{z}\right)+V_{\text {wake }}(z, \bar{\Psi}, \mathcal{J}),
$$

where the wake potential

$$
\begin{aligned}
V_{\text {wake }}= & \chi \mathcal{J} \int d \hat{\mathcal{Z}} F(\hat{\mathcal{Z}} ; s) W_{Q}^{\beta}(z-\hat{z}) \\
& +4 \chi \sqrt{\mathcal{J}} \cos \left(\bar{\Psi}-k_{\xi} z\right) \int d \hat{\mathcal{Z}} F(\hat{\mathcal{Z}} ; s) \\
& \times \sqrt{\hat{\mathcal{J}}} \cos \left(\hat{\bar{\Psi}}-k_{\xi} \hat{z}\right) W_{D}^{\beta}(z-\hat{z}) .
\end{aligned}
$$

For convenience we have defined the coupling constant $\chi$ and the total, beta function-weighted dipole and quadrupole wakefields as follows:

$$
\begin{gathered}
\chi \equiv \frac{e^{2} N_{e}}{2 \gamma m c^{2} \mathcal{C}_{R}}=\frac{I}{I_{A}} \frac{2 \pi}{\gamma Z_{0} c}, \\
W_{D, Q}^{\beta}(z-\hat{z}) \equiv \sum_{\ell} \beta_{x}^{\ell} W_{D, Q}^{\ell}(z-\hat{z}),
\end{gathered}
$$

where the average bunch current $I \equiv e c N_{e} / \mathcal{C}_{R}$, the Alfvén current $I_{A} \equiv 4 \pi \epsilon_{0} m c^{3} / e \approx 17 \mathrm{kA}$, and the impedance of free space $Z_{0} \equiv 1 /\left(\epsilon_{0} c\right) \approx 377 \Omega \quad\left(\epsilon_{0}\right.$ is the vacuum permittivity).

\section{B. Dissipative (Fokker-Planck) dynamics}

We have specified the conservative (Hamiltonian) part of Eq. (3), and now need to add the dissipative right-hand side. As mentioned before, this dissipative Fokker-Planck operator accounts for the stochastic emission of synchrotron radiation and for the subsequent energy replacement delivered by the rf cavities. We write the average change in mean energy, angle, and mean square energy as $\left\langle\Delta p_{z}\right\rangle,\left\langle\Delta x^{\prime}\right\rangle$, and $\left\langle\left(\Delta p_{z}\right)^{2}\right\rangle$, respectively, so that the dissipative evolution over one turn is (see Appendix A for more details)

$$
\begin{aligned}
\left.\frac{\partial F}{\partial s}\right|_{\mathrm{F} . \mathrm{P} .} \approx & -\frac{1}{\mathcal{C}_{R}} \oint d s \frac{\partial}{\partial p_{z}}\left[\frac{\left\langle\Delta p_{z}\right\rangle}{\Delta s} F(\mathcal{Z} ; s)\right]_{\mathrm{SR}+\mathrm{rf}} \\
& -\frac{1}{\mathcal{C}_{R}} \oint d s \frac{\partial}{\partial x^{\prime}}\left[\frac{\left\langle\Delta x^{\prime}\right\rangle}{\Delta s} F(\mathcal{Z} ; s)\right]_{\mathrm{rf}} \\
& +\frac{1}{\mathcal{C}_{R}} \oint d s \frac{\partial^{2}}{\partial p_{z}^{2}}\left[\frac{\left\langle\left(\Delta p_{z}\right)^{2}\right\rangle}{2 \Delta s} F(\mathcal{Z} ; s)\right]_{\mathrm{SR}} .
\end{aligned}
$$


The first two terms give the damping due to energy loss from synchrotron emission and its replacement by the rf-cavities, while the final term gives a diffusive spreading in energy due to the stochastic nature of synchrotron emission; we give a brief description of each of these below.

We first consider the average change in the mean energy due to synchrotron emission and its replenishment from the rf cavities over one turn. We will choose the rf to cancel the energy lost by the reference electron over one turn, so that the term $\propto\left\langle\Delta p_{z}\right\rangle$ results in a damping of the coordinates toward the reference orbit. As was shown in [11] (see also, e.g., $[23,31]$ ), the explicit expression for the mean energy change is

$$
\begin{aligned}
\frac{\left\langle\Delta p_{z}\right\rangle}{\Delta s}= & \frac{P_{\gamma}(s)}{\gamma_{0} m c^{3}}-\frac{V_{\mathrm{rf}}(s)}{\gamma_{0} m c^{2}} \\
\approx & \frac{P_{\gamma_{0}}(s)}{\gamma_{0} m c^{3}}-\frac{V_{\mathrm{rf}}(s)}{\gamma_{0} m c^{2}}-p_{z} \frac{2 P_{\gamma_{0}}(s)}{\gamma_{0} m c^{3}} \\
& +x \frac{P_{\gamma_{0}}(s)}{\gamma_{0} m c^{3}}\left[\frac{\kappa}{\rho(s)}+\frac{2}{B_{y}} \frac{\partial B_{y}}{\partial x}\right],
\end{aligned}
$$

where $V_{\mathrm{rf}}(s)$ is the energy gain per unit length from the rf, $P_{\gamma}(s)$ is the power emitted to synchrotron radiation by an electron of energy $\gamma$ at location $s, \rho$ is the bending radius, $B_{y}$ is the bending magnetic field, and $\kappa$ is a geometric quantity to be explained shortly. Stable motion requires the rf energy gain to match that lost due to radiation emission of the reference particle, so that $\oint d s\left(V_{\mathrm{rf}}-P_{\gamma_{0}} / c\right)=0$. The final term on the first line in (26) reflects the fact that higher energy electrons radiate more, which tends to damp the energy towards its reference value $\gamma_{0}$. The second line $\propto x$ is nonzero if the emission depends on the horizontal displacement, which may occur if the path length or field strength depends on $x$. The former effect is reflected by the purely geometric term $\kappa / \rho$ : for rectangular dipoles the path length is independent of $x$ and $\kappa=0$, while for sector magnets the path length through the dipole increases linearly with $x$ and $\kappa \rightarrow 1$ (the $\kappa$ for other magnet shapes can also be determined). The final term in (26) $\propto \partial B_{y} / \partial x$ allows for the field strength to vary transversely.

The second damping term in the Fokker-Planck operator (24) arises because synchrotron emission reduces the total electron energy, while the rf only replaces the longitudinal momentum component. This in turn leads to an effective damping of the electron angle given by [11]

$$
\frac{\left\langle\Delta x^{\prime}\right\rangle}{\Delta s} \approx x^{\prime} \frac{P_{\gamma_{0}}(s)}{\gamma_{0} m c^{3}} .
$$

Finally, the third term in Eq. (24) gives the mean square energy increase due to the uncertainty associated with photon emission. We have [11]

$$
\frac{\left\langle\left(\Delta p_{z}\right)^{2}\right\rangle}{\Delta s}=\frac{55}{48 \sqrt{3}} \frac{\alpha \hbar^{2} \gamma_{0}^{5}}{(m c)^{2}} \frac{1}{\rho(s)^{3}},
$$

where $\alpha$ is the fine structure constant and $\hbar$ is Planck's constant divided by $2 \pi$.

We have specified the Fokker-Planck operator in terms of $p_{z}, x$, and $x^{\prime}$; to apply it in Eq. (3) we need to transform all expressions into the action-angle coordinates of the betatron motion. Doing this requires inverting the coordinate relationships (6)-(7) into

$$
\begin{aligned}
\mathcal{J}= & \frac{1}{2 \beta_{x}}\left(x+\eta_{x} p_{z}\right)^{2} \\
+ & \frac{\beta_{x}}{2}\left[\left(x^{\prime}+\eta_{x}^{\prime} p_{z}\right)-\frac{\beta_{x}^{\prime}}{2 \beta_{x}}\left(x+\eta_{x} p_{z}\right)\right]^{2} \\
& \tan \Psi=-\beta_{x} \frac{x^{\prime}+\eta_{x}^{\prime} p_{z}}{x+\eta_{x} p_{z}}+\frac{\beta_{x}^{\prime}}{2},
\end{aligned}
$$

and transforming the derivatives using

$$
\begin{gathered}
\frac{\partial}{\partial p_{z}} \rightarrow \frac{\partial}{\partial p_{z}}+\frac{\partial \mathcal{J}}{\partial p_{z}} \frac{\partial}{\partial \mathcal{J}}+\frac{\partial \Psi}{\partial p_{z}} \frac{\partial}{\partial \Psi} \\
\frac{\partial}{\partial x^{\prime}} \rightarrow \frac{\partial \mathcal{J}}{\partial x^{\prime}} \frac{\partial}{\partial \mathcal{J}}+\frac{\partial \Psi}{\partial x^{\prime}} \frac{\partial}{\partial \Psi},
\end{gathered}
$$

where we have neglected a small chromatic correction to the last line coming from the transformation (16). Carrying out the coordinate transformation is a long exercise in applying the chain rule. In terms of the new (action-angle) coordinates, the resulting Fokker-Planck equation is (see, e.g., [23])

$$
\begin{aligned}
\frac{\partial F}{\partial s}+\{F, \mathcal{H}\}= & \mathfrak{D}_{z} \frac{\partial^{2} F}{\partial p_{z}^{2}}+\frac{2}{c \tau_{z}}\left(p_{z} \frac{\partial F}{\partial p_{z}}+F\right) \\
& +\mathfrak{D}_{x}\left(\mathcal{J} \frac{\partial^{2} F}{\partial \mathcal{J}^{2}}+\frac{\partial F}{\partial \mathcal{J}}+\frac{1}{4 \mathcal{J}} \frac{\partial^{2} F}{\partial \Psi^{2}}\right) \\
& +\frac{2}{c \tau_{x}}\left(\mathcal{J} \frac{\partial F}{\partial \mathcal{J}}+F\right) .
\end{aligned}
$$

Here we have neglected terms $\sim e^{ \pm 2 i \Psi}$ that oscillate at twice the betatron frequency, cross derivatives between the longitudinal and transverse plane that will result in a small modification of the equilibrium, and have defined $\tau_{z}$ and $\mathfrak{D}_{z}$ to be the usual longitudinal damping time and diffusion coefficient, respectively, with $\tau_{x}$ and $\mathfrak{D}_{x}$ their transverse counterparts. The inverse damping times are

$$
\begin{aligned}
& \frac{1}{\tau_{z}}=\frac{1}{\mathcal{C}_{R}} \oint d s \frac{P_{\gamma}(s)}{2 \gamma m c^{2}}\left[2+\eta_{x}\left(\frac{\kappa}{\rho}+\frac{2}{B_{x}} \frac{\partial B}{\partial x}\right)\right] \\
& \frac{1}{\tau_{x}}=\frac{1}{\mathcal{C}_{R}} \oint d s \frac{P_{\gamma}(s)}{2 \gamma m c^{3}}\left[1-\eta_{x}\left(\frac{\kappa}{\rho}+\frac{2}{B_{x}} \frac{\partial B}{\partial x}\right)\right],
\end{aligned}
$$


while the diffusion coefficients

$$
\begin{gathered}
\mathfrak{D}_{z}=\frac{55 \gamma^{5}}{48 \sqrt{3}} \frac{\alpha \hbar^{2}}{(m c)^{2}} \frac{1}{\mathcal{C}_{R}} \oint d s \frac{1}{\rho(s)^{3}} \\
\mathfrak{D}_{x}=\frac{55 \gamma^{5}}{48 \sqrt{3}} \frac{\alpha \hbar^{2}}{(m c)^{2}} \frac{1}{\mathcal{C}_{R}} \oint d s \frac{\beta_{x} \eta_{x}^{\prime 2}+2 \alpha_{x} \eta_{x} \eta_{x}^{\prime}+\gamma_{x} \eta_{x}^{2}}{\rho(s)^{3}} .
\end{gathered}
$$

In Eq. (37) we have introduced the usual Courant-Snyder (Twiss) parameters $\alpha_{x}$ and $\gamma_{x}$, with $\alpha_{x} \equiv-\beta_{x}^{\prime} / 2$ and $\gamma_{x} \beta_{x}=1+\alpha_{x}^{2}$; the integrand in (37) is often written as "curly $\mathcal{H}$ " [11].

Finally, we will find it convenient to rewrite Eq. (33) in terms of the following products of damping and diffusion coefficients,

$$
\sigma_{\delta}^{2} \equiv \frac{c \mathfrak{D}_{z} \tau_{z}}{2}, \quad \varepsilon_{0} \equiv \frac{c \mathfrak{D}_{x} \tau_{x}}{2} .
$$

We will show that $\sigma_{\delta}$ equals the equilibrium energy spread while $\varepsilon_{0}$ is the equilibrium emittance $\varepsilon_{0}$, as our choice of notation suggests. Then, the Fokker-Planck equation (33) becomes

$$
\begin{aligned}
\frac{\partial F}{\partial s}+\{F, \mathcal{H}\}= & \frac{2}{c \tau_{z}}\left[\sigma_{\delta}^{2} \frac{\partial^{2} F}{\partial p_{z}^{2}}+p_{z} \frac{\partial F}{\partial p_{z}}+F\right] \\
& +\frac{2}{c \tau_{x}}\left[\varepsilon_{0} \mathcal{J} \frac{\partial^{2} F}{\partial \mathcal{J}^{2}}+\frac{\varepsilon_{0}}{4 \mathcal{J}} \frac{\partial^{2} F}{\partial \Psi^{2}}\right. \\
& \left.+\left(\mathcal{J}+\varepsilon_{0}\right) \frac{\partial F}{\partial \mathcal{J}}+F\right]
\end{aligned}
$$

\section{Equilibrium and perturbed distributions}

The Hamiltonian left-hand side of (33) vanishes if the distribution function depends only on the conserved quantities of $\mathcal{H}$. For the unperturbed motion with $V_{\text {wake }}=0$, the two conserved quantities are the betatron action $\mathcal{J}$ and the longitudinal Hamiltonian $\mathcal{H}_{z}$. Furthermore, since the unperturbed $\mathcal{H}$ is separable we assume that $F_{0}$ is as well, and write the equilibrium distribution

$$
F_{0}\left(z, \bar{\Psi}, p_{z}, \mathcal{J} ; s\right)=g_{0}\left(\mathcal{H}_{z}\right) f_{0}(\mathcal{J}) .
$$

Inserting $F_{0}$ into the Fokker-Planck equation (33) leads to

$$
\begin{aligned}
0= & f_{0}\left[\frac{2}{c \tau_{z}} \frac{\partial}{\partial p_{z}}\left(p_{z} g_{0}\right)+\mathfrak{D}_{z} \frac{\partial^{2} g_{0}}{\partial p_{z}^{2}}\right] \\
& +g_{0}\left[\frac{2}{\tau_{x}} \frac{\partial}{\partial \mathcal{J}}\left(\mathcal{J} f_{0}\right)+\mathfrak{D}_{x}\left(\mathcal{J} \frac{\partial^{2} f_{0}}{\partial \mathcal{J}^{2}}+\frac{\partial f_{0}}{\partial \mathcal{J}}\right)\right] .
\end{aligned}
$$

The nontrivial solution requires both terms in square brackets to vanish, in which case it is straightforward to show that the equilibrium solution is a Gaussian in energy $p_{z}$ and a decaying exponential in action $\mathcal{J}$,

$$
\begin{aligned}
F_{0}\left(z, p_{z}, \mathcal{J}\right) & =\frac{\exp \left\{-\frac{1}{2 \sigma_{\delta}^{2}}\left[p_{z}^{2}+\frac{2}{\alpha_{c}} V(z)\right]\right\}}{2 \pi \sigma_{\delta} \sigma_{z}} \frac{\exp \left(-\frac{\mathcal{J}}{\varepsilon_{0}}\right)}{2 \pi \varepsilon_{0}} \\
& =\frac{\exp \left(-\mathcal{H}_{z} / \alpha_{c} \sigma_{\delta}^{2}\right)}{2 \pi \sigma_{\delta} \sigma_{z}} \frac{\exp \left(-\mathcal{J} / \varepsilon_{0}\right)}{2 \pi \varepsilon_{0}}
\end{aligned}
$$

To include the effects of wakefields, we now add to the equilibrium $F_{0}$ the perturbed distribution $F_{1}$. While the unperturbed distribution is the simple function of $\mathcal{J}$ and $\mathcal{H}_{z}$ given by Eq. (42), we will choose the perturbed distribution to model dipole oscillations of the (possibly unstable) beam. Since the longitudinal and transverse physics are largely uncoupled, we assume that both $F_{0}$ and $F_{1}$ are separable into their transverse and longitudinal parts, writing

$$
\begin{aligned}
F\left(z, \bar{\Psi}, p_{z}, \mathcal{J} ; s\right)= & f_{0}(\mathcal{J}) g_{0}\left(\mathcal{H}_{z}\right) \\
& +f_{1}(\bar{\Psi}, \mathcal{J} ; s) g_{1}\left(z, p_{z} ; s\right) .
\end{aligned}
$$

The transverse physics is dominated by the betatron oscillations that occur on a time scale much faster than any other dynamics considered here. Hence, it is reasonable to assume that the perturbation along $x$ is well described by a simple dipole making betatron oscillations, while all the wakefield-driven complexity appears in the (relatively) slowly evolving longitudinal direction. One can envision a sausage-like electron bunch that may flop around longitudinally due to wakefields as it executes its betatron oscillations. Following Sacherer [5], such a simple dipole in $x$ is given by

$$
f_{1}(\bar{\Psi}, \mathcal{J} ; s)=-\mathcal{D}(s) \sqrt{\frac{1}{2}} \mathcal{J} f_{0}^{\prime}(\mathcal{J}) e^{i \bar{\Psi}} e^{-i \omega_{\beta} s / c},
$$

which can be understood by computing the complex dipole moment

$$
\begin{aligned}
\int d x_{\beta} d x_{\beta}^{\prime} \frac{x_{\beta}+i \beta_{x} x_{\beta}^{\prime}}{\sqrt{\beta_{x}}} f_{1} & \sim \int d \Psi d \mathcal{J} \sqrt{2 \mathcal{J}} e^{-i \Psi} f_{1}(\Psi, \mathcal{J}) \\
& =\mathcal{D}(s) e^{-i \omega_{\beta} s / c}
\end{aligned}
$$

Whether or not the dipole amplitude $\mathcal{D}(s)$ increases or decreases will depend on the wakefields and on the beam current.

Furthermore, we will assume that the perturbed distribution is small and linearize the resulting equation, in which case $F_{1}$ can be expressed as a linear sum of oscillating modes $\propto e^{-i \Omega s / c}$, with $\Omega$ the complex frequency. Hence, we write the perturbed distribution 


$$
\begin{aligned}
F_{1} & =-\mathcal{D}(s) \sqrt{\frac{1}{2}} \mathcal{J} f_{0}^{\prime}(\mathcal{J}) e^{i \bar{\Psi}} e^{-i \omega_{\beta} s / c} g_{1}\left(z, p_{z} ; s\right) \\
& =-\sqrt{\frac{1}{2}} \mathcal{J} f_{0}^{\prime}(\mathcal{J}) e^{i \Psi} \tilde{g}_{1}\left(z, p_{z}\right) e^{-i \Omega s / c} e^{-i \omega_{\beta} s / c},
\end{aligned}
$$

where in the second line we isolate the time dependence by defining $\mathcal{D}(s) g_{1}\left(z, p_{z} ; s\right)=\tilde{g}_{1}\left(z, p_{z}\right) e^{-i \Omega s / c}$. Note that $\Omega$ represents the frequency difference in the oscillation from the betatron frequency, and the chromatic dependence is contained in $\bar{\Psi}$.

We can now specify the wakefield potential (21) in terms of the distribution function (43), using $F_{0}$ and $F_{1}$ given by Eqs. (42) and (46), respectively. Upon inserting $F$ into the expression for $V_{\text {wake }}$ we obtain

$$
\begin{aligned}
V_{\text {wake }}= & \chi \mathcal{J} \int d \hat{z} d \hat{p}_{z} g_{0}\left(\hat{z}, \hat{p}_{z}\right) W_{Q}^{\beta}(z-\hat{z}) \\
& +\chi \sqrt{2 \mathcal{J}} \cos \left(\bar{\Psi}-k_{\xi} z\right) e^{-i\left(\Omega+\omega_{\beta}\right) s / c} \\
& \times \int d \hat{z} d \hat{p}_{z} \tilde{g}_{1}\left(\hat{z}, \hat{p}_{z}\right) e^{i k_{\xi} \hat{z}} W_{D}^{\beta}(z-\hat{z}) .
\end{aligned}
$$

Note that only the perturbed distribution contributes to the dipolar term, since the kick due to $W_{D}$ is proportional to the displacement of the source electron. The quadrupolar term, on the other hand, scales with the test electron action $\mathcal{J}$ and with the number of driving electrons $\sim F_{0}$.

\section{Linearized equation for the transverse motion}

We have assumed that the perturbed distribution performs simple betatron oscillations along $x$, which has led to the prescribed transverse dependence of $F_{1}$ given by Eq. (46). Hence, we can now integrate out the transverse degrees of freedom to obtain an equation for the longitudinal perturbation $\tilde{g}_{1}$. Operationally, we will evaluate the dipole motion by multiplying Eq. (39) by $\sqrt{2 \mathcal{J}} e^{-i \bar{\Psi}}$ and integrating over $\bar{\Psi}$ and $\mathcal{J}$. Only those terms of the equation that originally vary as $\sim e^{i \bar{\Psi}}$ will survive this integration. Using the wakefield (47) and the distribution function defined by Eqs. (42) and (46), the Hamiltonian part of (39) that oscillates near the betatron frequency is

$$
\begin{aligned}
\frac{\partial F}{\partial s}+\{F, \mathcal{H}\}= & -\frac{i\left(\Omega+\omega_{\beta}\right)}{c} F_{1}+\frac{\omega_{\beta}}{c} \frac{\partial F_{1}}{\partial \bar{\Psi}} \\
& +\frac{\partial V_{\text {wake }}^{Q}}{\partial \mathcal{J}} \frac{\partial F_{1}}{\partial \bar{\Psi}}-\frac{\partial V_{\text {wake }}^{D}}{\partial \bar{\Psi}} \frac{\partial F_{0}}{\partial \mathcal{J}} \\
& +\left\{F_{1}, \mathcal{H}_{z}\right\}+\cdots,
\end{aligned}
$$

where we use "..." to denote those terms that do not oscillate $\sim e^{i \bar{\Psi}}$, are nonlinear in $\tilde{g}_{1}$, or are otherwise small. In particular, the force due to the wakefields results by neglecting the second line in

$$
\begin{aligned}
\left\{F_{1}, V_{\text {wake }}\right\}= & \frac{\partial V_{\text {wake }}^{Q}}{\partial \mathcal{J}} \frac{\partial F_{1}}{\partial \bar{\Psi}}-\frac{\partial V_{\text {wake }}^{D}}{\partial \bar{\Psi}} \frac{\partial F_{1}}{\partial \mathcal{J}} \\
& +\left\{F_{1}, V_{\text {wake }}^{D}\right\}-\frac{\partial V_{\text {wake }}}{\partial z} \frac{\partial F_{1}}{\partial p_{z}}
\end{aligned}
$$

since $\left\{F_{1}, V_{\text {wake }}^{D}\right\}$ is nonlinear in $F_{1}$ while the second term is a factor $\varepsilon_{x} / \sigma_{z} \sigma_{\delta} \ll 1$ smaller than those of the first line.

Now, we insert (48) and the expression for $F_{1}$ Eq. (46) into the Fokker-Planck equation (39), multiply by $i \sqrt{2 \mathcal{J}} e^{-i \bar{\Psi}} e^{i\left(\Omega+\omega_{\beta}\right) s / c}$ and integrate over $\bar{\Psi}$ to get

$$
\begin{aligned}
& -\mathcal{J} f_{0}^{\prime}\left[\left(\frac{\Omega}{c}-\chi \int d \hat{z} d \hat{p}_{z} g_{0} W_{Q}^{\beta}\right) \tilde{g}_{1}\right. \\
& \left.-\chi g_{0} \int d \hat{z} d \hat{p}_{z} \tilde{g}_{1} e^{i k_{\xi}(\hat{z}-z)} W_{D}^{\beta}+i\left\{\tilde{g}_{1}, \mathcal{H}_{z}\right\}\right] \\
& =-\mathcal{J} f_{0}^{\prime} \frac{2}{c \tau_{z}}\left[\sigma_{\delta}^{2} \frac{\partial^{2} \tilde{g}_{1}}{\partial p_{z}^{2}}+p_{z} \frac{\partial \tilde{g}_{1}}{\partial p_{z}}+\tilde{g}_{1}\right] \\
& +\mathcal{J} f_{0}^{\prime} \frac{i}{c \tau_{x}} \tilde{g}_{1}-\frac{2 i}{c \tau_{x}} \frac{\partial}{\partial \mathcal{J}}\left(\varepsilon_{0} \mathcal{J}^{2} f_{0}^{\prime \prime}+\mathcal{J}^{2} f_{0}^{\prime}\right) \tilde{g}_{1} .
\end{aligned}
$$

Here, we have rewritten the transverse derivatives so that the next integration over $\mathcal{J}$ is trivial if we use the fact that $-\mathcal{J} f_{0}^{\prime}=-d\left(\mathcal{J} f_{0}\right)+f_{0}$ and that $\int d \mathcal{J} f_{0}=1 / 2 \pi$. Hence, integrating out the transverse degree of freedom implies that the lowest order dipole varies longitudinally with a perturbation $\tilde{g}_{1}$ that obeys

$$
\begin{gathered}
{\left[\frac{\Omega+i / \tau_{x}}{c}-\chi \int d \hat{p}_{z} d \hat{z} W_{Q}^{\beta}(z-\hat{z}) g_{0}\left(\hat{z}, \hat{p}_{z}\right)\right] \tilde{g}_{1}} \\
-\chi g_{0} \int d \hat{p}_{z} d \hat{z} W_{D}^{\beta}(z-\hat{z}) e^{i k_{\xi}(\hat{z}-z)} \tilde{g}_{1}\left(\hat{z}, \hat{p}_{z}\right) \\
+i\left\{\tilde{g}_{1}, \mathcal{H}_{z}\right\}=\frac{2 i}{c \tau_{z}}\left[\sigma_{\delta}^{2} \frac{\partial^{2} \tilde{g}_{1}}{\partial p_{z}^{2}}+p_{z} \frac{\partial \tilde{g}_{1}}{\partial p_{z}}+\tilde{g}_{1}\right] .
\end{gathered}
$$

There are several interesting points that we would like to make regarding the linearized equation (51). First, we find that the transverse dissipative dynamics reduces to betatron damping such that $\Omega \sim-i / \tau_{x}$ in the $\chi \rightarrow 0$ limit. This simplification is due to the assumed perturbation $f_{1}$ : Eq. (44) describes rigid transverse dipole oscillations whose shape in transverse phase-space is left invariant by diffusion. Hence, only a damping term remains after we integrate out the transverse degree of freedom. This should be contrasted with the right-hand side of (51), which reflects both damping and diffusion due to dynamics in the longitudinal plane.

The second thing to note about (51) is that quadrupolar wakefield alone cannot lead to an instability. In fact, if $W_{D}$ were to hypothetically vanish, we could eliminate $W_{Q}$ from (51) entirely by making the replacement 


$$
\tilde{g}_{1}\left(z, p_{z}\right) \rightarrow \tilde{\tilde{g}}_{1}\left(z, p_{z}\right) e^{-i \chi s \int d \hat{p}_{z} d \hat{z} W_{Q}^{\beta}(z-\hat{z}) g_{0}\left(\hat{z}, \hat{p}_{z}\right)}
$$

in the perturbation Eq. (46). We can understand this physically because in the linear limit $W_{Q}$ acts much like a quadrupole magnet on any individual electron, giving rise to only a current-dependent tune shift. Third, we find that the head-tail phase appears in the dipolar wakefield term. Writing the wakefield as an impedance via the Fourier transform gives

$$
\begin{aligned}
W_{D}^{\beta}(z-\hat{z}) e^{i k_{\xi}(\hat{z}-z)} & =-\frac{i c}{2 \pi} \int d k Z_{D}^{\beta}(k) e^{i k(z-\hat{z})} e^{i k_{\xi}(\hat{z}-z)} \\
& =\int d k \frac{c Z_{D}^{\beta}(k)}{2 \pi i} e^{i\left(k-k_{\xi}\right)(z-\hat{z})},
\end{aligned}
$$

so that the frequencies that contribute to the dipolar impedance are effectively shifted by the head-tail frequency $c k_{\xi}$. We will see later that this can be used to stabilize lower-order modes by increasing the chromaticity, thereby increasing the beam current at which transverse collective instabilities occur.

In the next section we turn to solving (51) when $\mathcal{H}_{z}$ describes the simple harmonic motion of a usual rf system. Our solution will make use of Sacherer's formalism to find the linear eigenmodes and complex eigenfrequencies associated with Eq. (51).

\section{LINEAR MODES FOR SIMPLE HARMONIC RF FOCUSING}

We take the longitudinal Hamiltonian to be that of the linear rf focusing,

$$
\mathcal{H}_{z}=\mathcal{H}_{\text {synch }}=\frac{\alpha_{c}}{2} p_{z}^{2}+\frac{\omega_{s}^{2}}{2 \alpha_{c} c^{2}} z^{2},
$$

where $\omega_{s}$ is the synchrotron frequency. This Hamiltonian describes simple harmonic motion, and we will find it convenient to introduce longitudinal action-angle coordinates just as we did for the transverse motion. Again, we make the transformation $\left(z, p_{z}\right) \rightarrow(\Phi, \mathcal{I})$ using the "typeone" mixed-variable generating function

$$
S_{1}^{\mathcal{I}}(\Phi, z)=-\frac{\omega_{s}^{2} z^{2}}{2 \alpha_{c} c} \tan \Phi,
$$

with $\mathcal{I}=-\partial S_{1}^{\mathcal{I}} / \partial \Phi$ and $p_{z}=\partial S_{1}^{\mathcal{I}} / \partial z$; we obtain the familiar expressions

$$
z=\sqrt{\frac{2 \mathcal{I} \alpha_{c} c}{\omega_{s}}} \cos \Phi \quad p_{z}=-\sqrt{\frac{2 \mathcal{I} \omega_{s}}{\alpha_{c} c}} \sin \Phi,
$$

so that the synchrotron Hamiltonian $\mathcal{H}_{z}=\omega_{s} \mathcal{I} / c$. Using Eq. (42) for $F_{0}$, we find that the longitudinal equilibrium for simple harmonic motion is uniform in angle and decreases exponentially with action,

$$
g_{0}(\mathcal{I})=\frac{\alpha_{c} c \sigma_{\delta}^{2}}{2 \pi \omega_{s}} e^{-\omega_{s} \mathcal{I} / \alpha_{c} c \sigma_{\delta}^{2}} \equiv \frac{e^{-\mathcal{I} /\langle\mathcal{I}\rangle}}{2 \pi\langle\mathcal{I}\rangle},
$$

where we have defined the average longitudinal action

$$
\langle\mathcal{I}\rangle=\frac{\alpha_{c} c}{\omega_{s}} \sigma_{\delta}^{2}=\frac{\omega_{s}}{\alpha_{c} c} \sigma_{z}^{2}=\sigma_{z} \sigma_{\delta}
$$

Now that the linear synchrotron Hamiltonian has specified the longitudinal equilibrium (57), we can simplify the quadrupolar term from (51). In particular, we write

$$
\begin{aligned}
\int d \hat{p}_{z} d \hat{z} g_{0} W_{Q}^{\beta}= & \int d \hat{\mathcal{I}} d \hat{\Phi} g_{0}(\mathcal{I}) \int d k \frac{c Z_{Q}^{\beta}(k)}{2 \pi i} e^{i k(z-\hat{z})} \\
= & \int d k \frac{c Z_{Q}^{\beta}(k)}{2 \pi i} e^{i k z} \\
& \times \int d \hat{r} d \hat{\Phi} \frac{e^{-\hat{r}}}{2 \pi} \sum_{\ell} i^{-\ell} J_{\ell}\left(k \sigma_{z} \sqrt{2 \hat{r}}\right) e^{i \ell \hat{\Phi}} \\
= & \frac{c}{2 \pi i} \int d k Z_{Q}^{\beta}(k) e^{i k z} e^{-k^{2} \sigma_{z}^{2} / 2}
\end{aligned}
$$

where the second line uses the scaled action $r \equiv \mathcal{I} /\langle\mathcal{I}\rangle$, expresses $\hat{z}$ in terms of action-angle variables and $\sigma_{z}$ using (56) and (58), and applies the Jacobi-Anger identity

$$
e^{i x \cos \Phi}=\sum_{\ell} i^{\ell} J_{\ell}(x) e^{i \ell \Phi},
$$

with $J_{\ell}(x)$ the Bessel function of order $\ell$. The final expression Eq. (59) obtains from the $p=m=0$ case of the integral [32]

$$
\int_{0}^{\infty} d x e^{-x} J_{m}(2 y \sqrt{x}) x^{m / 2} L_{p}^{m}(x)=\frac{1}{p !} y^{2 p+m} e^{-y^{2}},
$$

where $L_{p}^{m}(x)$ is the associated Laguerre polynomial; this integral will prove useful later as well.

We also must express the Fokker-Planck diffusion and damping operators in terms of the action-angle variables. Convenient expressions can be found in terms of the scaled action $r \equiv \mathcal{I} /\langle\mathcal{I}\rangle$; for example, derivatives with respect to $p_{z}$ become

$$
\begin{aligned}
\frac{\partial}{\partial p_{z}} & =-\sqrt{\frac{2 \mathcal{I} \alpha_{c} c}{\omega_{s}}} \sin \Phi \frac{\partial}{\partial \mathcal{I}}-\sqrt{\frac{\alpha_{c} c}{2 \mathcal{I} \omega_{s}}} \cos \Phi \frac{\partial}{\partial \Phi} \\
& =-\frac{\sqrt{2 r}}{\sigma_{\delta}} \sin \Phi \frac{\partial}{\partial r}-\frac{1}{\sigma_{\delta} \sqrt{2 r}} \cos \Phi \frac{\partial}{\partial \Phi} .
\end{aligned}
$$

Using Eqs. (56), (59), and (62), the linearized FokkerPlanck equation (51) becomes 


$$
\begin{aligned}
& {\left[\frac{\Omega+i / \tau_{x}}{c}+\frac{i \omega_{s}}{c} \frac{\partial}{\partial \Phi}+\frac{i c \chi}{2 \pi} \int d k Z_{Q}^{\beta}(k) e^{-k^{2} \sigma_{z}^{2} / 2} e^{i k z}\right] \tilde{g}_{1}} \\
& \quad+\frac{i c \chi}{2 \pi} g_{0} \int d \hat{\mathcal{I}} d \hat{\Phi} \tilde{g}_{1}(\hat{\Phi}, \hat{\mathcal{I}}) \int d k Z_{D}^{\beta}(k) e^{i\left(k-k_{\xi}\right)(z-\hat{z})} \\
& =\frac{2 i}{c \tau_{z}}\left[r \frac{\partial^{2}}{\partial r^{2}}+(r+1) \frac{\partial}{\partial r}+1+\frac{4 r+1}{4 r} \frac{\partial^{2}}{\partial \Phi^{2}}\right] \tilde{g}_{1} \\
& \quad-\left\{\frac{i e^{2 i \Phi}}{c \tau_{z}}\left[r \frac{\partial^{2}}{\partial r^{2}}+r \frac{\partial}{\partial r}+i \frac{\partial^{2}}{\partial r \partial \Phi}+\frac{1-r}{2 i} \frac{\partial}{\partial \Phi}-\frac{1}{4 r} \frac{\partial^{2}}{\partial \Phi^{2}}\right]\right. \\
& \quad \text { c.c. }\} \tilde{g}_{1} .
\end{aligned}
$$

where our notation is such that $z=z(\Phi, \mathcal{I})$ and $\hat{z}=z(\hat{\Phi}, \hat{\mathcal{I}})$.

We solve (63) by expanding the perturbation $\tilde{g}_{1}$ as a series of orthogonal, linear modes. Since $\tilde{g}_{1}$ is periodic in $\Phi$ the angular dependence is given by the Fourier harmonics $e^{i n \Phi}$, and we expand the perturbation as

$$
\begin{aligned}
\tilde{g}_{1}(\Phi, \mathcal{I}) & =\sum_{q, n} a_{q}^{n} g_{q}^{n}(\mathcal{I}) g_{0}(\mathcal{I}) e^{i n \Phi} \\
& =\sum_{q, n} a_{q}^{n} g_{q}^{n}(\mathcal{I}) \frac{e^{-\mathcal{I} /\langle\mathcal{I}\rangle}}{2 \pi\langle\mathcal{I}\rangle} e^{i n \Phi} .
\end{aligned}
$$

Here, $a_{q}^{n}$ is the mode coefficient while $g_{q}^{n}(\mathcal{I})$ is the mode function for the action (radial) mode $q$ and angular (azimuthal) mode $n$. We now insert the angular expansion (64) into the linear equation (63). First, we use the Bessel expansion (60) to rewrite the $z$ dependence in the quadrupolar wakefield term as

$$
\begin{aligned}
& \frac{i c \chi}{2 \pi} \tilde{g}_{1}(\Phi, \mathcal{I}) \int d k Z_{Q}^{\beta}(k) e^{-k^{2} \sigma_{z}^{2} / 2} e^{i k z} \\
& =\frac{i c \chi}{2 \pi} \sum_{q, n} a_{q}^{n} g_{q}^{n}(\mathcal{I}) g_{0}(\mathcal{I}) e^{i n \Phi} \\
& \quad \times \int d k Z_{Q}^{\beta}(k) e^{-k^{2} \sigma_{z}^{2} / 2} \sum_{\ell} i^{\ell} J_{\ell}\left(k \sigma_{z} \sqrt{2 r}\right) e^{i \ell \Phi} .
\end{aligned}
$$

Next, inserting the angular decomposition (64) into the integral in the dipolar wakefield term gives

$$
\begin{aligned}
& \int d k d \hat{\mathcal{I}} d \hat{\Phi} Z_{D}^{\beta}(k) e^{i\left(k-k_{\xi}\right)(z-\hat{z})} \tilde{g}_{1} \\
& =\int d k Z_{D}^{\beta}\left(k+k_{\xi}\right) e^{i k z} \int d \hat{r} d \hat{\Phi} \sum_{q, n} a_{q}^{n} g_{q}^{n}(\hat{r}) \frac{e^{-\hat{r}}}{2 \pi} e^{i n \hat{\Phi}} \\
& \quad \times \sum_{\ell} i^{\ell} J_{-\ell}\left(k \sigma_{z} \sqrt{2 \hat{r}}\right) e^{i \ell \hat{\Phi}} \\
& =\int d k Z_{D}^{\beta}\left(k+k_{\xi}\right) \sum_{\ell} i^{\ell} J_{\ell}\left(k \sigma_{z} \sqrt{2 r}\right) e^{i \ell \Phi} \\
& \quad \times \sum_{q, n} \frac{a_{q}^{n}}{i^{n}} \int d \hat{r} g_{q}^{n}(\hat{r}) J_{n}\left(k \sigma_{z} \sqrt{2 \hat{r}}\right) e^{-\hat{r}} .
\end{aligned}
$$

We insert (65) and (66) into (51), multiply by $e^{-i m \Phi}$, and integrate over angle, to find that the left-hand (Hamiltonian) side of the mode equation (63) becomes

$$
\begin{aligned}
& \sum_{q} \frac{a_{q}^{m}}{c}\left(\Omega-m \omega_{s}+i / \tau_{x}\right) g_{q}^{m}(r) e^{-r} \\
& \quad+\frac{i c \chi}{2 \pi} \int d k Z_{Q}^{\beta}(k) e^{-k^{2} \sigma_{z}^{2} / 2} \\
& \quad \times \sum_{q, n} \frac{a_{q}^{n}}{i^{n-m}} J_{m-n}\left(k \sigma_{z} \sqrt{2 r}\right) g_{q, n}(r) e^{-r} \\
& \quad+\frac{i c \chi}{2 \pi} \int d k Z_{D}^{\beta}(k) J_{m}\left[\left(k-k_{\xi}\right) \sigma_{z} \sqrt{2 r}\right] e^{-r} \\
& \quad \times \sum_{q, n} \frac{a_{q}^{n}}{i^{n-m}} \int d \hat{r} J_{n}\left[\left(k-k_{\xi}\right) \sigma_{z} \sqrt{2 \hat{r}}\right] g_{q}^{n}(\hat{r}) e^{-\hat{r}}
\end{aligned}
$$

In the limit $r \rightarrow 0$, the dipole wakefield term implies that $g_{q}^{m}(r) \sim J_{m}(\sqrt{r}) \sim r^{|m| / 2}$, which is consistent with the quadrupole wakefield contribution. As emphasized in Ref. [8], the $r \rightarrow 0$ behavior singles out a unique set of basis functions $g_{q}^{m}(r)$. In our case, it implies that the mode functions associated with the angular dependence $e^{i m \Phi}$ must form a complete basis for functions that behave as $r^{|m| / 2}$ at small $r$. To satisfy this small $r$ limit, we specify the modes to be given by the Gauss-Laguerre functions as follows:

$$
\begin{aligned}
\tilde{g}_{1}(\Phi, r) & =\sum_{q, n} a_{q}^{n} g_{q}^{n}(r) \frac{e^{-r}}{2 \pi} e^{i n \Phi} \\
& =\sum_{q=0}^{\infty} \sum_{n=-q}^{\infty} a_{q}^{n} \frac{r^{n / 2} L_{q}^{n}(r)}{\sqrt{(q+n) ! / q !}} \frac{e^{-r}}{2 \pi} e^{i n \Phi} .
\end{aligned}
$$

The angular expansion over the range $-q \leq n<\infty$ appears to be a departure from the previous work in Ref. [10], where the Fourier sum was assumed to run over all integer $n$, and each term in the summation had $n$ replaced by $|n|$. It turns out that one can show that the two expansions are equivalent, but we have found that our representation is simpler to manipulate; for an example we refer the reader Appendix B, where we apply Eq. (68) to the decoherence of a kicked beam.

Regardless, these technical details do become important when we consider dynamics at large chromaticity, where stability depends largely on the higher order modes. In this case it is also important to completely calculate the FokkerPlanck contributions. As we show in Appendix A, these dissipative terms can be written in a much simpler way than that presented in Ref. [10]; after a somewhat lengthy calculation, we find that 


$$
\begin{aligned}
& \left\{r \frac{\partial^{2}}{\partial r^{2}}+(r+1) \frac{\partial}{\partial r}+1+\frac{4 r+1}{4 r} \frac{\partial^{2}}{\partial \Phi^{2}}\right. \\
& \quad-\left[\frac { e ^ { 2 i \Phi } } { 2 } \left(r \frac{\partial^{2}}{\partial r^{2}}+r \frac{\partial}{\partial r}+i \frac{\partial^{2}}{\partial r \partial \Phi}\right.\right. \\
& \left.\left.\left.\quad+\frac{1-r}{2 i} \frac{\partial}{\partial \Phi}-\frac{1}{4 r} \frac{\partial^{2}}{\partial \Phi^{2}}\right)- \text { c.c. }\right]\right\} \tilde{g}_{1} \\
& =\sum_{n, q} a_{q}^{n} \frac{r^{n / 2} e^{-r}}{\sqrt{(q+n) ! / q !}} \frac{e^{i n \Phi}}{2 \pi}\left[-\left(q+\frac{n}{2}\right) L_{q}^{n}(r)\right. \\
& \left.\quad+e^{2 i \Phi} r L_{q-1}^{n+2}(r)+e^{-2 i \Phi} \frac{(n+q)(q+1)}{r} L_{q+1}^{n-2}(r)\right] .
\end{aligned}
$$

As we did with the Hamiltonian part, we eliminate the angular dependence by multiplying Eq. (63) by $e^{-i m \Phi}$ and integrating over $\Phi$, finding that the dissipative right-hand side of Eq. (63) becomes

$$
\begin{aligned}
& \int_{0}^{2 \pi} d \Phi \frac{e^{-i m \Phi}}{2 \pi} \frac{2 i}{c \tau_{z}}\left[\sigma_{\delta}^{2} \frac{\partial^{2} \tilde{g}_{1}}{\partial p_{z}^{2}}+p_{z} \frac{\partial \tilde{g}_{1}}{\partial p_{z}}+\tilde{g}_{1}\right] \\
& =-\frac{i}{c \tau_{z}} \sum_{q} \frac{r^{m / 2} e^{-r} L_{q}^{m}(r)}{\sqrt{(q+m) ! / q !}}(2 q+m) a_{q}^{m} \\
& +\frac{i}{c \tau_{z}} \sum_{q} \frac{r^{m / 2} e^{-r} L_{q-1}^{m}(r)}{\sqrt{(q+m-2) ! / q !}} a_{q}^{m-2} \\
& +\frac{i}{c \tau_{z}} \sum_{q} \frac{r^{m / 2} e^{-r} L_{q+1}^{m}(r)}{\sqrt{(q+m+2) ! / q !}}(q+1)(q+m+1) a_{q}^{m+2}
\end{aligned}
$$

Now, we have all the necessary pieces to write down a solution to Eq. (63), namely, the Hamiltonian left-hand side given by Eq. (67), and the dissipative right-hand side Eq. (70). We isolate the mode coefficient $a_{p}^{m}$ by multiplying both sides by $r^{m / 2} L_{p}^{m}(r)$, integrating over $r$, and using the orthogonality relation

$$
\int_{0}^{\infty} d r r^{m} e^{-r} L_{p}^{m}(r) L_{q}^{m}(r)=\frac{(p+m) !}{p !} \delta_{p, q}
$$

We further simplify the resulting equation by applying the integral (61), and then write the resulting linear mode equation as

$$
\begin{aligned}
& {\left[\Omega-m \omega_{s}+\frac{i}{\tau_{x}}+\frac{i}{\tau_{z}}(2 p+m)\right] a_{p}^{m}} \\
& \quad+\sum_{n, q}(\mathrm{D}+\mathrm{Q})_{p, q}^{m, n} a_{q}^{n}=i\left(R_{p}^{m} a_{p+1}^{m-2}+T_{p}^{m} a_{p-1}^{m+2}\right) .
\end{aligned}
$$

Here we have defined the coupling matrix associated with the dipole wakefield as

$$
\begin{aligned}
\mathrm{D}_{p, q}^{m, n}= & \frac{i c I}{I_{A}} \frac{i^{m-n}}{\gamma Z_{0}} \int d k Z_{D}^{\beta}\left(k+k_{\xi}\right) e^{-k^{2} \sigma_{z}^{2}} \\
& \times \frac{\left(k \sigma_{z} / \sqrt{2}\right)^{2 p+m}}{\sqrt{p !(p+m) !}} \frac{\left(k \sigma_{z} / \sqrt{2}\right)^{2 q+n}}{\sqrt{q !(q+n) !}},
\end{aligned}
$$

that from the quadrupolar wakefield as

$$
\begin{aligned}
\mathrm{Q}_{p, q}^{m, n}= & \frac{i c I}{I_{A}} \frac{i^{n-m}}{\gamma Z_{0}} \sqrt{\frac{p !}{(p+m) !} \frac{q !}{(q+n) !}} \\
& \times \int d k Z_{Q}^{\beta}(k) e^{-k^{2} \sigma_{z}^{2} / 2} \int_{0}^{\infty} d y e^{-y} y^{(n+m) / 2} \\
& \times J_{n-m}\left(k \sigma_{z} \sqrt{2 y}\right) L_{p}^{m}(y) L_{q}^{n}(y),
\end{aligned}
$$

and the diffusive coupling terms

$$
\begin{aligned}
& R_{p}^{m}=\frac{1}{2 \tau_{z}} \sqrt{(p+1)(p+m)}, \\
& T_{p}^{m}=\frac{1}{2 \tau_{z}} \sqrt{p(p+m+1)} .
\end{aligned}
$$

Equation (72) is a matrix equation for the linear orthogonal modes $a_{p}^{m}$, and solving it numerically requires truncating the equation at some finite number of modes $N_{\text {mode }}$; since each $a_{p}^{m}$ is indexed by both $p$ and $m$, this involves picking a set of $(p, m)$ pairs that approximately spans the unstable solution. Then, Eq. (72) becomes an $N_{\text {mode }} \times N_{\text {mode }}$ matrix equation for the vectors $a_{p}^{m}$, which can be solved for the $N_{\text {mode }}$ eigenvalues and eigenvectors. The instability threshold current $I_{\text {thresh }}$ is defined by the smallest $I$ for which any $\Omega$ gets a positive imaginary part. We have found that $N_{\text {mode }}$ increases as $\xi_{x}$ increases both because one needs to include more radial modes (indexed by $p$ ), and because one needs to extend the range of azimuthal modes to more negative $m$.

Perhaps the first property evident from (72) is the fact that the mode of radial order $p$ and azimuthal number $m$ has an effective damping rate given by

$$
\left.\frac{1}{\tau}\right|_{p, m}=\frac{1}{\tau_{x}}+\frac{2 p+m}{\tau_{z}} .
$$

This mode-dependent damping was first derived in Ref. [10], although to our knowledge the full consequences of Eq. (76) have not been entirely appreciated. First, it implies that the small amplitude decoherence of a kicked beam depends on the chromaticity as we show in Appendix B. Second and more importantly, this mode-dependent damping becomes crucial for properly calculating collective effects at large positive chromaticity when the higher-order modes dictate transverse stability. Physically, the enhanced "damping" of higher-order modes comes about because diffusion smooths out shorter-wavelength perturbations faster than those that 
vary over larger distances. The last consequence of the Fokker-Planck operator is in the coupling terms (75). These terms are smaller than the diagonal damping, and we have found that including these off-diagonal terms typically increases the maximum stable current by a few to several percent. Regardless, the terms $R$ and $T$ are easy enough to include.

Finally, the dipole and quadrupole wakefields couple the modes together through the matrices $D$ and $Q$, respectively. To represent real wakefields the imaginary parts of $Z_{Q}^{\beta}(k)$ and $Z_{D}^{\beta}(k)$ must be symmetric functions of $k$, while the real parts are antisymmetric. This implies that the quadrupolar matrix $Q$ is purely real, while the dipolar $D$ is complex unless the chromaticity vanishes so that $k_{\xi}=0$. Furthermore, the quadrupolar matrix is symmetric, $\mathrm{Q}_{p, q}^{m, n}=\mathrm{Q}_{q, p}^{n, m}$, so that $\mathrm{Q}$ is a real, symmetric matrix with purely real eigenvalues.

\section{COMPARISON WITH TRACKING}

In this section we compare predictions of the FokkerPlanck theory Eq. (72) with simulations using the particle tracking code ELEGANT and its parallel version PELEGANT $[15,33]$. We use the multi-bend achromat (MBA) upgrade lattice planned for the Advanced Photon Source as our model storage ring [16], whose lattice functions and relevant parameters are summarized in Fig. 1. We track particles through the ring using the ILMATRIX element in ELEGANT, which allows for fast, symplectic particle tracking through a periodic cell including chromatic and amplitude-dependent tunes, beta functions, and dispersion. ILMATRIX does this by computing a linear matrix for each particle that is determined both by user-supplied parameters and the particle coordinates. The user-supplied quantities include the Twiss parameters, tunes, dispersion, etc, and how these quantities depend on the particle energy (giving chromatic effects through third order in $p_{z}$ ) and on the transverse coordinates (which provide the nonlinear terms in $\mathcal{J}$ ). We have found that the ILMATRIX element provides a fast and reasonably accurate way to track particles through a wide variety of lattices.
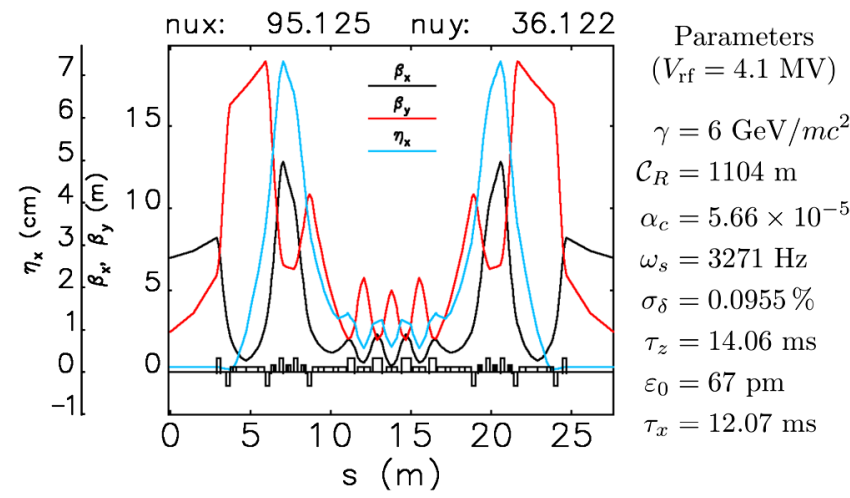

FIG. 1. Lattice Twiss functions and parameters used in the tracking simulations.
Since the ILMATRIX element allows for higher-order chromatic and nonlinear terms in the Hamiltonian, our tracking can potentially include more physics then does our theoretical model. We have found numerically that including the dependence of the tune on the oscillation amplitude does not affect the instability threshold for either the present APS or the MBA upgrade lattice, but does change the large amplitude oscillation dynamics. In other words, for our parameters effects of Landau damping are negligible, and we expect them to be similarly small for most high-energy electron storage rings. On the other hand, the MBA's large second order chromaticity is an important effect that can reduce transverse instability thresholds by $20 \%$ or more. The simulations shown here artificially set the second order chromaticity to zero, and we plan to address its inclusion in a subsequent paper.

We complete our tracking simulation by modeling the acceleration from the rf cavity, the damping and diffusion due to synchrotron emission, and the effects of impedance as lumped elements applied once per turn; within ELEGANT these correspond to the RFCA, SREFFECTS, and ZTRANSERSE elements, respectively. For our studies here we will take a simple model of the transverse impedance, assuming that its source is limited to the resistive wall of the vacuum chamber. Specifically, we assume that the chamber is either round or essentially flat with a half-gap of $b(s)$ that varies slowly over its length. Then, the ring-average dipole impedance can be approximated by

$$
Z_{D}^{\beta}(k)=\eta_{D} \oint d s \beta_{x}(s) \frac{\operatorname{sgn}(k)-i}{\pi b(s)^{3}} \sqrt{\frac{Z_{0} \rho(s)}{2|k|}},
$$

where $\rho(s)$ is the piecewise constant resistivity, $\operatorname{sgn}(k)$ gives the sign of $k$, and the factor $\eta_{D}$ depends on the chamber geometry, with $\eta_{D}=1$ for round chambers and $\eta_{D}=\pi^{2} / 24$ for flat chambers (see, e.g, [34,35]).

We list the lengths, minimum half-gap, and resistivity of the various vacuum chambers planned for the MBA in Table I. Note that for most of the ring the half-gap $b(s)$ is constant at either its nominal value of $11 \mathrm{~mm}$ or at the minimum half-gap $b=3 \mathrm{~mm}$ in each of the $35,5-\mathrm{m}$ long insertion devices (IDs). The one exception is the transition pieces to and from the IDs, where we take $b(s)$ to vary

TABLE I. Resistive wall impedance elements.

\begin{tabular}{lcccc}
\hline \hline Element & $\left\langle\beta_{x}\right\rangle(\mathrm{m})$ & $b(\mathrm{~mm})$ & $\rho(\Omega \mathrm{m})$ & $L(\mathrm{~m})$ \\
\hline Flat IDs & 7 & 3 & $3.16 \times 10^{-8}$ & 150 \\
Round IDs & 7 & 3 & $3.16 \times 10^{-8}$ & 25 \\
ID transition & 8 & $11 \rightarrow 3$ & $3.16 \times 10^{-8}$ & 6.3 \\
ID transition & 8 & $3 \rightarrow 11$ & $3.16 \times 10^{-8}$ & 6.3 \\
Al chamber & 10 & 11 & $3.16 \times 10^{-8}$ & 605 \\
Cu chamber & 3 & 11 & $1.68 \times 10^{-8}$ & 224 \\
SS 314L chamber & 9 & 11 & $95.2 \times 10^{-8}$ & 85 \\
\hline \hline
\end{tabular}


linearly over the $0.18 \mathrm{~m}$ length. This distinction is not crucial, since the transitions contribute $\sim 2 \%$ of the total resistive wall impedance, while the small-gap IDs account for about $70 \%$ of $Z_{D}^{\beta}$. We can summarize all this by saying that we take the scaled, $\beta$ function-weighted dipolar impedance to be

$Z_{D}^{\beta}(k)=Z_{\mathrm{RW}} \frac{\operatorname{sgn}(k)-i}{|k[1 / \mathrm{m}]|^{1 / 2}} \quad$ with $\quad Z_{\mathrm{RW}}=25 \mathrm{M} \Omega$.

Finally, while the quadrupolar impedance vanishes in round vacuum chambers, the flat ID chambers have $Z_{Q}(k)=-Z_{D}(k)$; we add this effect in Sec. IV C.

\section{A. Transverse mode coupling in the low-chromaticity limit}

The limit of zero chromaticity has been studied extensively in the past, and we only briefly review the relevant physics. For $\xi_{x}=0$ the impedance matrix $\mathrm{D}$ becomes purely real, and it turns out that stability is almost entirely dictated by the modes with indices $(p=0, m=0)$ and $(p=1, m=-1)$. In this case the matrices are $2 \times 2$ and closed form solutions can be found. Furthermore, all the Fokker-Planck damping and diffusive terms are small since $\omega_{s} \gg 1 / \tau_{z}, 1 / \tau_{x}$, and the instability mechanism is welldescribed by the usual transverse mode coupling instability (TMCI) theory [17-20]. As we show in Fig. 2(a), the impedance shifts the (approximately real) frequency of the $(0,0)$ mode towards that of the $(0,-1)$ mode, and an instability arises when the two frequencies "merge" and the two eigenfrequencies become (approximate) complex conjugates of each other at $I_{\text {thresh }} \approx 0.52 \mathrm{~mA}$.

While the two-mode theory continues to be valid at small chromaticity, the mode coupling picture just described becomes less accurate as $\xi_{x}$ grows. We show in Fig. 2(b) the dependence of $\Omega$ on the current when $\xi_{x}=0.75$. Although Fig. 2(b) indicates that the general behavior of $\Omega(I)$ is similar to the case of vanishing chromaticity, the beam becomes unstable at $I_{\text {thresh }} \approx 0.39 \mathrm{~mA}$ without any obvious merging of $\mathfrak{R}(\Omega)$ that would indicate mode coupling.

Finally, we compare the predicted instability threshold current of the two-mode theory to that found in simulations in Fig. 2(c). At zero chromaticity we see that doubling the rf voltage increases $I_{\text {thresh }}$ by a factor $1.2 \approx 2^{1 / 4}$. This is because the instability arises when the impedance shifts the frequency of the $(0,0)$ mode by an amount of order $\omega_{s} \propto V_{\mathrm{rf}}^{1 / 2}$. Since the strength of the resistive wall impedance scales as

$$
\begin{aligned}
\mathrm{D} & \sim \int d k \frac{e^{-k^{2} \sigma_{z}^{2}}}{|k|^{1 / 2}}\left(k \sigma_{z} / \sqrt{2}\right)^{2(p+q)+m+n} \\
& \propto \frac{1}{\sigma_{z}^{1 / 2}} \int d x \frac{e^{-2 x^{2}}}{|x|^{1 / 2}} x^{2(p+q)+m+n} \propto V_{\mathrm{rf}}^{1 / 4},
\end{aligned}
$$
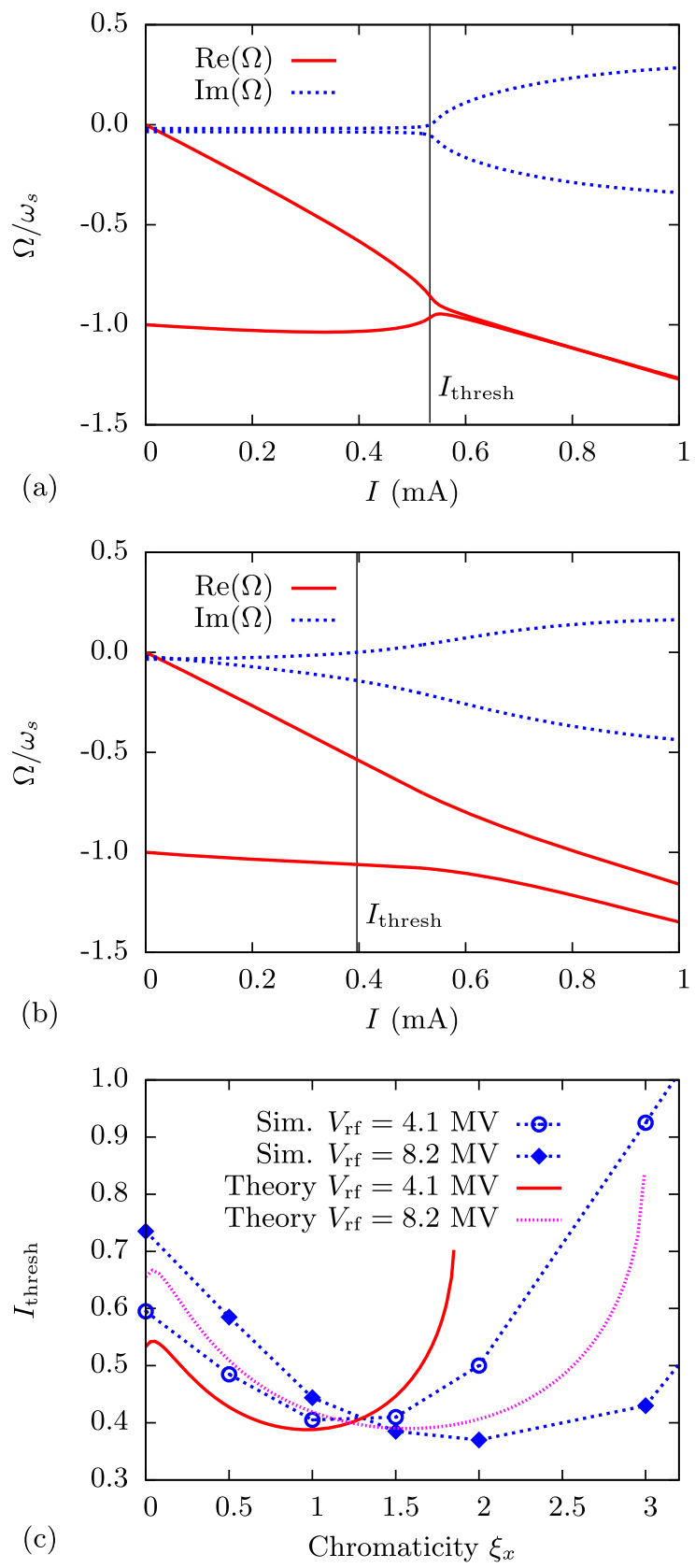

FIG. 2. Real and imaginary parts of $\Omega$ for the two-mode theory with $\xi_{x}=0$ (a) and $\xi_{x}=0.75$ (b). Panel (c) compares the instability threshold predicted by theory and simulation for two different rf voltages. The two mode theory follows the theory reasonably well provided $k_{\xi} \sigma_{z}=2 \pi \xi_{x} \sigma_{z} / \alpha_{c} \mathcal{C}_{R} \lesssim 0.7$.

the current at which the instability occurs scales as $V_{\mathrm{rf}}^{1 / 4}$. Note that this statement depends on the impedance; if the wakefield is constant behind the driving charge $Z_{D}^{\beta}(k) \sim 1 / k$ and $I_{\text {thresh }} \sim \omega_{s} \sim V_{\text {rf }}^{1 / 2}[7,8]$.

For small but nonzero chromaticity, Fig. 2(c) shows that the instability threshold current first decreases and then increases. While the specific shape of this curve will inevitably depend on the precise form of $Z_{D}$, the initial drop 
in $I_{\text {thresh }}$ is related to the fact that $Z_{D}\left(k+k_{\xi}\right)$ is complex for $k_{\xi} \neq 0$. When the chromaticity becomes sufficiently large the two-mode approximation fails, as it predicts that the beam is stable at any current when $\xi_{x}>1.9$ for $V_{\text {rf }}=4.1 \mathrm{MV}$, and $\xi_{x}>3$ for $V_{\mathrm{rf}}=8.2 \mathrm{MV}$. For both voltages the two-mode approximation ceases to be valid when the rms head-tail phase $k_{\xi} \sigma_{z} \gtrsim 0.7$. Hence, we conclude that the two-mode approximation applies in general only if $k_{\xi} \sigma_{z} \lesssim 1$. For higher values of $\xi_{x}$ the unstable solution is comprised of a more complicated superposition of modes, and requires solving the problem with matrices that are larger than $2 \times 2$. Furthermore, in this regime the unstable modes include terms with relatively high radial and azimuthal mode numbers $p$ and $m$, in which case predicting transverse collective instability requires including the Fokker-Planck damping and coupling terms. We compare theory and simulation at arbitrary values of $\xi_{x}$ in the next section.

\section{B. Transverse instabilities at high chromaticity}

As previously mentioned, when $\xi_{x} \sigma_{z} \gtrsim 1$ the reduced model comprised of two modes becomes stable. In this case one must enlarge the matrices of Eq. (72) to include higherorder azimuthal and radial modes, and we have found that the matrix size required to obtain convergence increases with $\xi_{x}$; for the largest chromaticity examples presented here we found that $\sim 500$ modes were needed.

We plot in Fig. 3(a) the instability threshold current as a function of chromaticity for two different $\mathrm{rf}$ voltages. Figure 3(a) shows that the Fokker-Planck theory closely follows the simulation results over a wide range of $\xi_{x}$. When $k_{\xi} \sigma_{z} \gtrsim 1$ the threshold current increases with $\xi_{x}$, and the longer bunch (smaller voltage) has a larger $I_{\text {thresh }}$ for a given chromaticity. In fact, when $k_{\xi} \sigma_{z} \gtrsim 1$ both simulation and theory indicate that $I_{\text {thresh }}$ is approximately a function of $k_{\xi} \sigma_{z}$ alone. As we show in Fig. 3(b), plotting $I_{\text {thresh }}$ as a function of $\xi_{x} \sigma_{z}$ results in the curves from Fig. 3(a) collapsing onto each other. Here, we only plot the simulation results from Fig. 3(a) as the solid red and dashed blue lines for clarity. The other two green and black lines labeled $W_{\text {const }}$ use the same lattice but assume that the wakefield is constant behind the exciting charge. This seems to indicate that $I_{\text {thresh }}$ may be approximately a function of only $k_{\xi} \sigma_{z}$ for other impedances as well, provided $k_{\xi} \sigma_{z} \gtrsim 1$.

An entirely different theoretical approach to predicting single-bunch collective instabilities at large chromaticity was also reported in Ref. [21]. This so-called "post headtail" theory is based on Laclare's formulation of singlebunch collective effects [36], and was shown to agree quite well with measurements at the ESRF over a wide range of (relatively large) chromaticity. A big advantage of the post head-tail theory is that the instability threshold current can be calculated using an attractively simple (though approximate) formula. However, attempts to compare our simulations to the formula in Ref. [21] were not very successful.
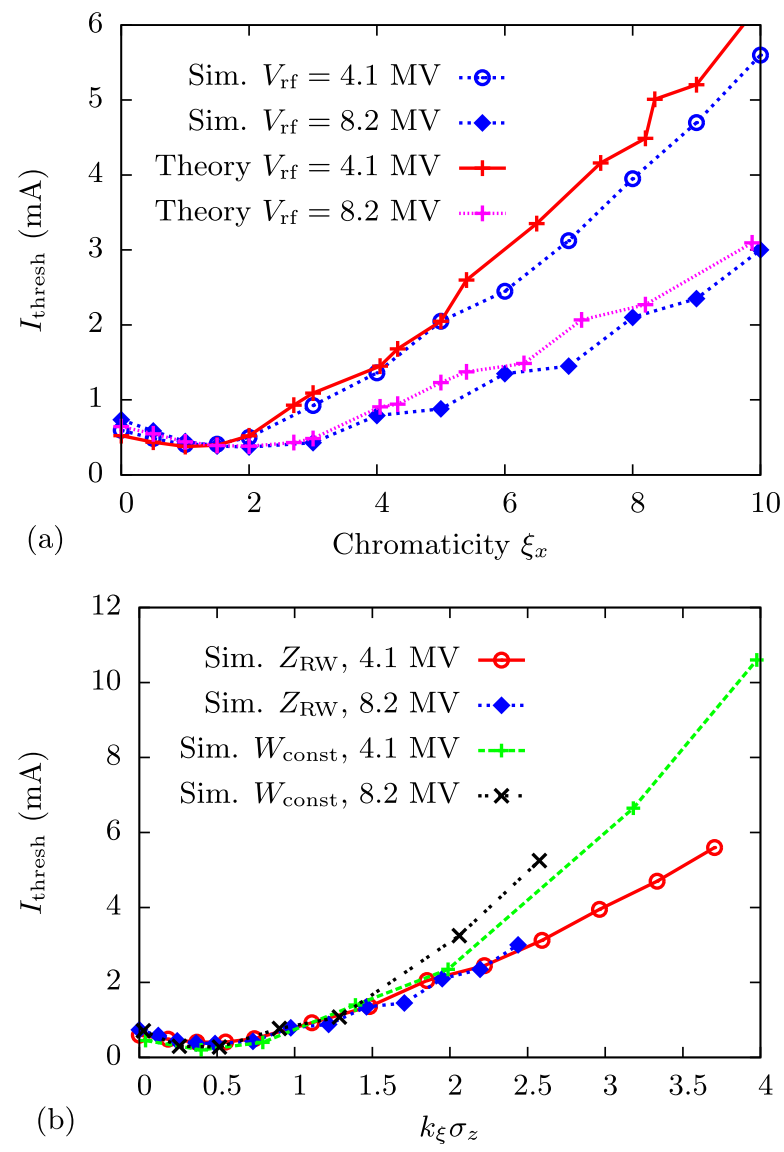

FIG. 3. (a) Maximum stable current $I_{\text {thresh }}$ as a function of chromaticity for two different rf voltages. As the chromatic headtail shift becomes larger than unity the current threshold is higher for the lower rf voltage that has a longer bunch length. (b) shows that the $I_{\text {thresh }}$ is approximately a function of $k_{\xi} \sigma_{z}$ for both the resistive wall impedance $Z_{\mathrm{RW}}$ and the constant wake $W_{\text {const }}$

We found that although the post head-tail instability threshold appears to have the correct qualitative dependence on lattice and impedance parameters, its slope with $\xi_{x}$ is smaller than that shown in Fig. 3(a) by about a factor of two. At this point we are not sure if the discrepancy arises from a misapplication of their theory, from a failure of some of the simplifying approximations, or from something else. For example, the simple formula in Ref. [21] is based on a broad-band resonator impedance model, while we focus on the resistive wall. In addition, we have found that the projection onto $z$ of the unstable mode observed in simulations only somewhat resembles the "shaker-mode" assumed in Ref. [21]. Hence, at present it appears that further work must be done to assess whether the post headtail theory may apply to our present study.

Not only does the theory predict the instability threshold current derived from tracking simulations, but it also accurately describes the unstable mode profile in longitudinal phase space. We demonstrate this in Fig. 4, where we compare the real (left) and imaginary (right) parts of $\tilde{g}_{1}$ 


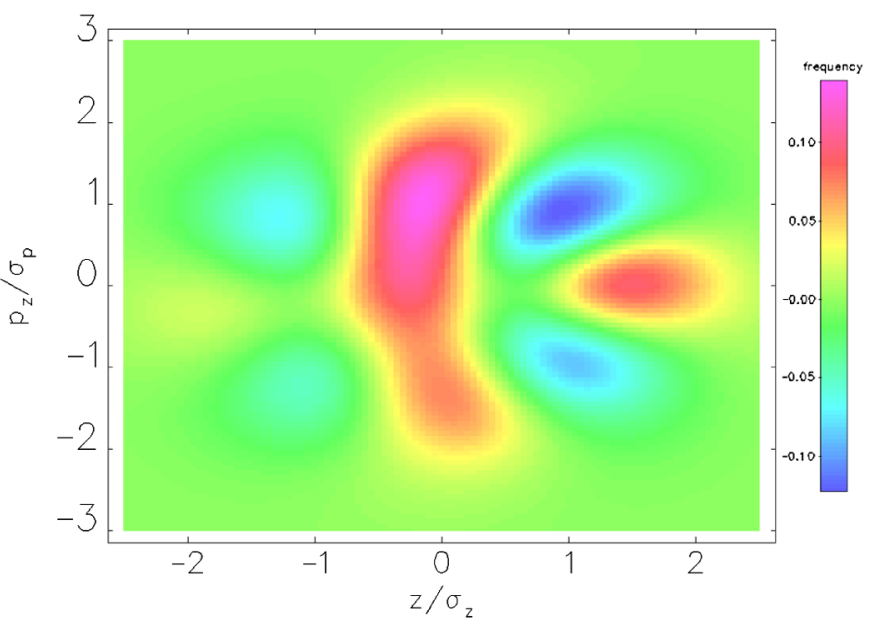

(a) Real part of $g_{1}$ from theory at $\xi=5$

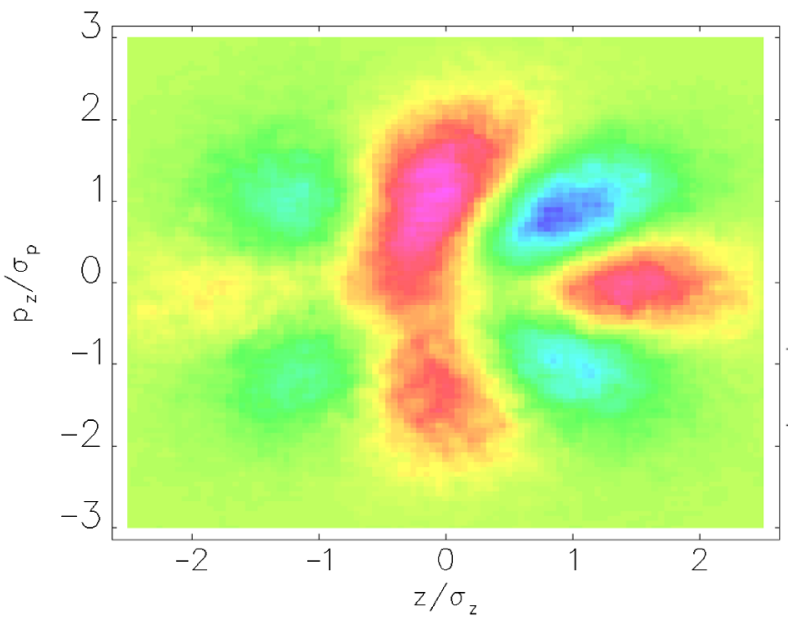

(c) Real part of $g_{1}$ from simulation at $\xi=5$

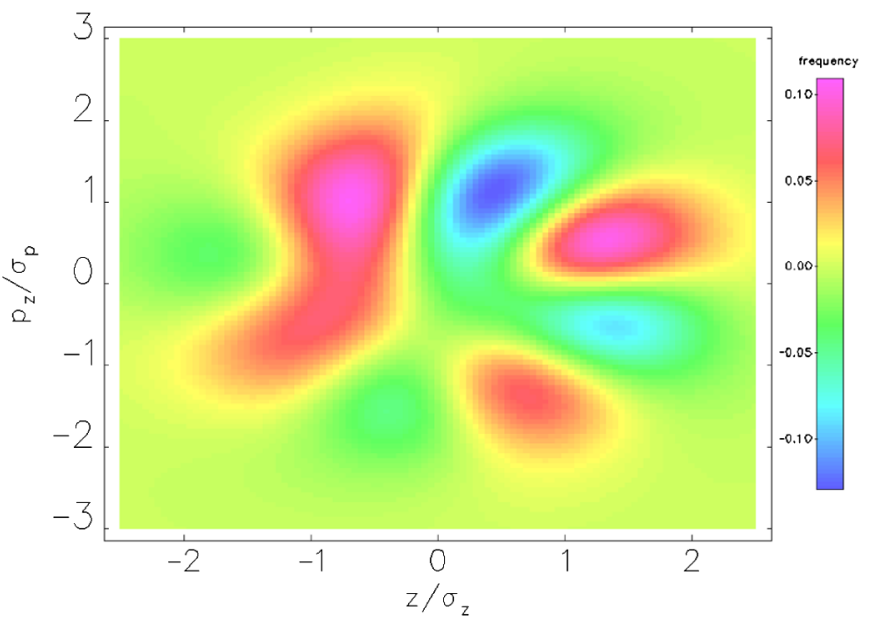

(b) Imaginary part of $g_{1}$ from theory at $\xi=5$

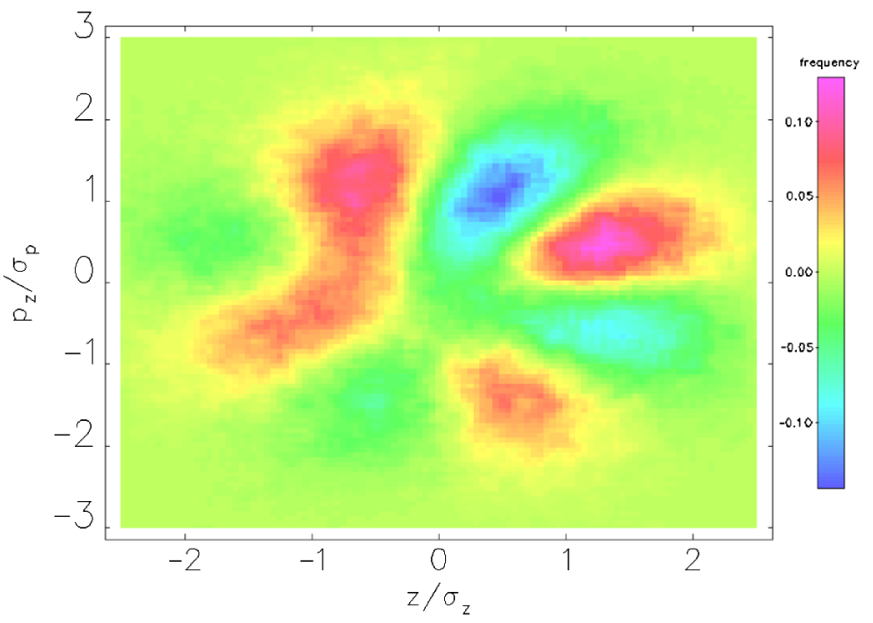

(d) Imaginary part of $g_{1}$ from simulation at $\xi=5$

FIG. 4. Comparison of the real and imaginary parts of the longitudinal distribution function $\tilde{g}_{1}$ obtained theoretically [(a) and (b)] and via simulation [(c) and (d)] at a chromaticity of 5 units. As indicated by the four pairs of lobes, the growing mode is dominated by the azimuthal mode number $m=-4$; theory indicates that $\sim 70 \%$ of the mode content has $m=-4$, while $\sim 20 \%$ has $m=-2, m=-3$, or $m=-4$. These plots map the variation in the longitudinal plane of the unstable betatron oscillation; at this turn in the simulation the betatron amplitude is about twice the rms beam size at equilibrium, while the maximum longitudinal variation is a little less than $\sigma_{x} / 2$.

computed using the matrix theory (top) with that obtained from ELEGANT simulations (bottom). The parameters for this comparison are $V_{\mathrm{rf}}=4.1 \mathrm{MV}, \xi_{x}=5$, and a single bunch current $I=2.25 \mathrm{~mA}>I_{\text {thresh }} \approx 2.05 \mathrm{~mA}$. The agreement is quite remarkable: both show the approximate azimuthal symmetry associated with the $m=-4$ mode, while more detailed features indicate other modal components. The theory predicts that $\sim 70 \%$ of $\tilde{g}_{1}$ is comprised of Gauss-Laguerre modes with $m=-4$, while the $m=-3$, $m=-5$, and $m=-2$ azimuthal components account for $9 \%, 6 \%$, and $5 \%$ of the unstable solution, respectively.

To properly interpret the phase portraits in Fig. 4, recall that they map out how the unstable betatron motion varies in the longitudinal plane. Hence, red indicates electrons that on average have a larger transverse displacement then the mean, while blue denotes a locally smaller betatron oscillation amplitude. In addition, if one views these phase protraits over many successive turns as a movie, the main lobes appear to approximately rotate clockwise at the synchrotron frequency, and the pattern shown in Fig. 4 repeats approximately every quarter synchrotron period. However, this "rotation" of the real and imaginary parts is not rigid, since the pass number dependence $e^{-i \Omega s}$ mixes the real and imaginary parts of $\tilde{g}_{1}$.

We obtained the simulation plots in Fig. 4(c) and (d) by extracting the perturbed distribution $\tilde{g}_{1}$ from the particle coordinates using its definition (46); operationally, we make a weighted histogram of all $N_{\text {sim }}$ simulation particles in longitudinal phase space, where the weight on the $j$ th particle is

$$
\frac{\sqrt{2 \mathcal{J}_{j}} e^{-i \bar{\Psi}_{j}} e^{i \omega_{\beta} s / c}}{N_{\operatorname{sim}}(\Delta z)\left(\Delta p_{z}\right)}=\frac{x_{j}+i \beta_{x} x_{j}^{\prime}}{N_{\operatorname{sim}} \sqrt{\beta_{x}}} \frac{e^{i\left(\omega_{\beta} s / c-k_{\xi} z_{j}\right)}}{(\Delta z)\left(\Delta p_{z}\right)},
$$


and $\Delta z$ and $\Delta p_{z}$ are the bin sizes in $z$ and $p_{z}$, respectively. This gives $\tilde{g}_{1}$ up to a constant complex factor. We eliminate its magnitude by dividing by the mean betatron oscillation amplitude $\langle\sqrt{2 \mathcal{J}}\rangle$, and find the phase by applying this procedure for many successive turns and choosing the best match. Finally, we have applied a simple nearest-neighbor smoothing to reduce the particle ganularity from the simulation-derived $\tilde{g}_{1}$.

\section{Effect of transverse quadrupolar impedance}

We have shown that the Fokker-Planck theory makes predictions that agree quite well with ELEGANT simulation results if we assume that the transverse collective effects are due to a purely dipolar impedance $Z_{D}$. In this section we show that the theory also performs well when we include the quadrupolar impedance $Z_{Q}$ due to chambers that are not axially symmetric. Again, we limit our discussion to the resistive wall impedance of round and essentially flat chambers, in which case the horizontal quadrupolar impedance $Z_{Q}=0$ and $Z_{Q}=-Z_{D}$, respectively (the vertical impedance $Z_{Q, y}=-Z_{Q, x}$ ). Then, we can write that the total impedance $Z_{Q}^{\beta}(k)=r_{Q} Z_{D}^{\beta}(k)$, with $\left|r_{Q}\right| \leq 1$. As was pointed out in Ref. [22], this form of the quadrupolar impedance can cancel some of the impedance-induced frequency shift of $\Re(\Omega)$, so that the mode coupling instability occurs at higher current.

We begin by analyzing the small chromaticity limit where the stability analysis can be approximated with two modes and analytic solutions can be found. These expressions become particularly compact when $\xi_{x}=0$ and $\omega_{s} \gg 1 / \tau_{x, z}$, in which case we drop the damping terms from (72) and all the $2 \times 2$ matrices are purely real. Computing the matrix elements of $D$ and $Q$, we find that two-mode stability equation of TMCI is

$$
\left[\begin{array}{cc}
\Omega+A_{0}\left(1+r_{Q}\right) & A_{1}\left(1+r_{Q}\right) \\
-A_{1}\left(1-r_{Q}\right) & \Omega+\omega_{s}+A_{0} \frac{1+7 r_{Q}}{8}
\end{array}\right]\left[\begin{array}{c}
a_{0}^{0} \\
a_{1}^{-1}
\end{array}\right]=0,
$$

where $r_{Q}$ if the ratio between the quadrupolar and dipolar resistive wall impedance, and we have defined

$$
A_{0} \equiv \frac{\Gamma\left(\frac{1}{4}\right) I}{\gamma I_{A}} \frac{c Z_{\mathrm{RW}}}{Z_{0} \sqrt{\sigma_{z}}}, \quad A_{1} \equiv \frac{\Gamma\left(\frac{3}{4}\right) I}{\gamma I_{A}} \frac{c Z_{\mathrm{RW}}}{Z_{0} \sqrt{2 \sigma_{z}}} .
$$

Solving (81) for $\Omega$ is simple enough, as is determining the condition on $I$ such that the beam is stable. Although not physically relevant, it turns out that $\Omega$ is always real and there is no instability if $\left|r_{Q}\right| \geq 1$. More realistically we have $\left|r_{Q}\right|<1$, in which case we have an instability when

$$
A_{0} \geq \frac{8 \Gamma\left(\frac{1}{4}\right) \omega_{s}}{\left(7+r_{Q}\right) \Gamma\left(\frac{1}{4}\right)+8 \sqrt{2\left(1-r_{Q}^{2}\right)} \Gamma\left(\frac{3}{4}\right)} .
$$
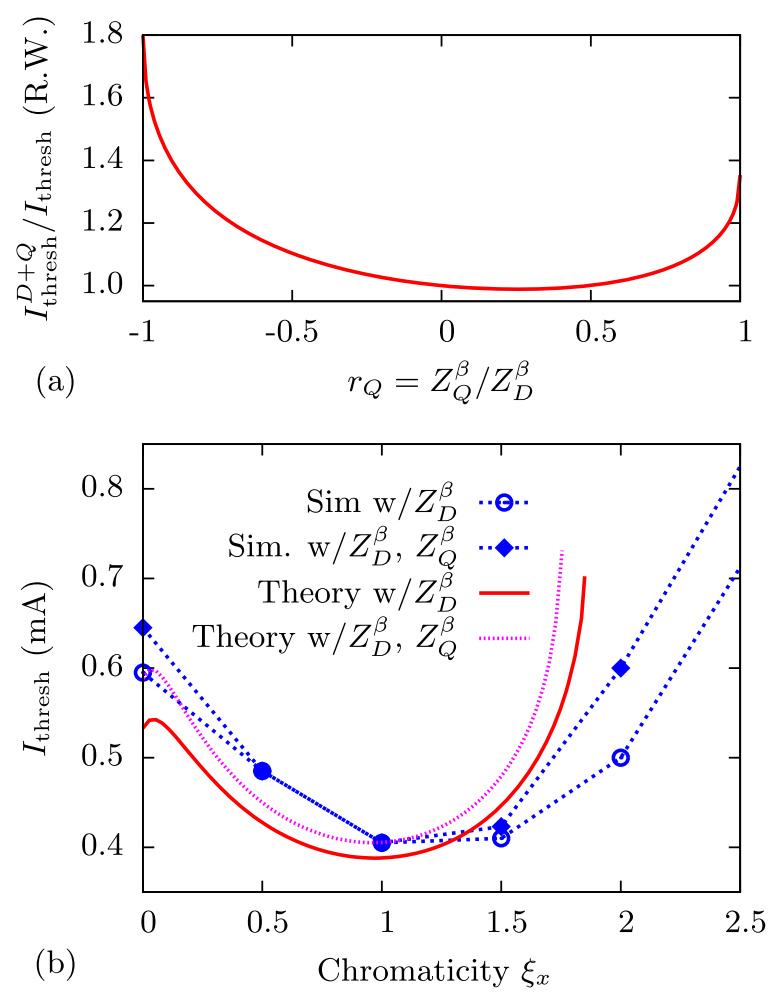

FIG. 5. (a) Change in the resistive wall instability threshold current at $\xi_{x}=0$ as a function of the quadrupolar to dipolar impedance ratio $r_{Q}$. (b) Comparison of the two-mode theory and simulation for the purely dipolar impedance $Z_{D}$ and the sum $Z_{D}+Z_{Q}$

If we write the ratio of the instability threshold current including the quadrupolar impedance to that without we find that

$$
\left.\frac{I_{\text {thresh }}^{D+Q}}{I_{\text {thresh }}}\right|_{\mathrm{RW}}=\frac{7 \Gamma\left(\frac{1}{4}\right)+8 \sqrt{2} \Gamma\left(\frac{3}{4}\right)}{\left(7+r_{Q}\right) \Gamma\left(\frac{1}{4}\right)+8 \sqrt{2\left(1-r_{Q}^{2}\right)} \Gamma\left(\frac{3}{4}\right)} .
$$

We plot this ratio in Fig. 5(a). For the specific case of the resistive wall elements listed in Table I, we have $Z_{D}=-Z_{Q}$ for the $150 \mathrm{~m}$ of flat ID chambers, and $Z_{Q}=0$ otherwise. This implies that the MBA has a ring-summed, betafunction-weighted impedance $Z_{Q}^{\beta}(k) \approx-Z_{D}^{\beta}(k) / 2$, and that including $Z_{Q}$ increases the $\xi_{x}=0$ instability threshold current by about $10 \%$.

We show how the instability threshold changes at small chromaticity in Fig. 5(b). Here we compare simulation results with the predictions of the complex two-mode theory. At zero chromaticity both theory and simulation indicate that $I_{\text {thresh }}$ is about $10 \%$ larger when $Z_{Q}$ is included, and we again find that at small but nonzero chromaticity $\left(0<k_{\xi} \sigma_{z} \lesssim 0.5\right) I_{\text {thresh }}$ decreases with $\xi_{x}$. For chromaticities larger than $\sim 1.6$ the two mode theory again fails, and we must include higher order modes and all the terms of the Fokker-Planck theory. 
We plot the stability threshold current over a wide range of chromaticity in Fig. 6(a), where the theory sets $Z_{Q}^{\beta} \approx$ $-Z_{D}^{\beta} / 2$ and allows for many radial and azimuthal modes (up 20-25 for each). Here we see that the quadrupolar impedance has a sizeable impact on the stability threshold once $k_{\xi} \sigma_{z} \gtrsim 2$, corresponding to $\xi_{x}>3.5$ here; Fig. 6(a) shows that the quadrupolar impedance increases $I_{\text {limit }}$ by $10 \%-40 \%$ in both the simulation and theory.

We conclude our discussion of collective instabilities including $Z_{Q}$ by comparing a theoretically predicted unstable mode profile to that derived from tracking. We choose the same $\xi_{x}=5$ as that of Fig. 4, although we now increase the single-bunch current to $I=2.9 \mathrm{~mA}>I_{\text {thresh }} \approx 2.75 \mathrm{~mA}$. The agreement in mode profiles in Fig. 6(b) and (c) is reasonably good, although not quite as impressive as before. For the unstable distributions shown in Fig. 6, three quarters of the Gauss-Laguerre basis functions are nearly evenly divided between $m=-4$ (at 35\%) and $m=-3$ (with 30\%), while the remaining modes are scattered over several different values of $m$. In general, we have found that the quadrupolar impedance results in unstable modes whose basis functions are more evenly divided over various azimuthal mode numbers.

\section{Effect of the longitudinal impedance}

The previous two sections have shown that the FokkerPlanck theory closely agrees with ELEGANT simulations provided the longitudinal potential is given entirely by a single frequency rf system with $V_{z}=V_{\text {synch }} \propto z^{2}$, which leads to a longitudinal equilibrium that is Gaussian in position and energy. In this section we investigate to what degree a longitudinal impedance may be incorporated into the theory. Adding a longitudinal impedance gives rise to a distortion of $V_{z}$ that depends on the current profile of the bunch. In this case the equilibrium distribution function must also be self-consistent with the total $V_{z}$, so the situation is significantly more complicated. Nevertheless, our Fokker-Planck theory can be extended to approximately include a longitudinal impedance $Z_{\|}$provided its resulting $V_{z}$ is only a small perturbation.

The longitudinal impedance results in a currentdependent distortion of $V_{z}$ that lengthens the bunch and (possibly) increases the energy spread. We can incorporate these effects within our model by taking

$$
\omega_{s} \rightarrow \omega_{s}(I)=\alpha_{c} \frac{c \sigma_{\delta}(I)}{\sigma_{z}(I)} .
$$

Hence, we expect this extension to fail at some current $I$ that depends on the longitudinal impedance. For our study here we continue to use the resistive wall $Z$, with $Z_{\|} \propto k b^{2} Z_{D}$; more precisely, we use the parameters of Table I in the formula for the ring-averaged longitudinal impedance
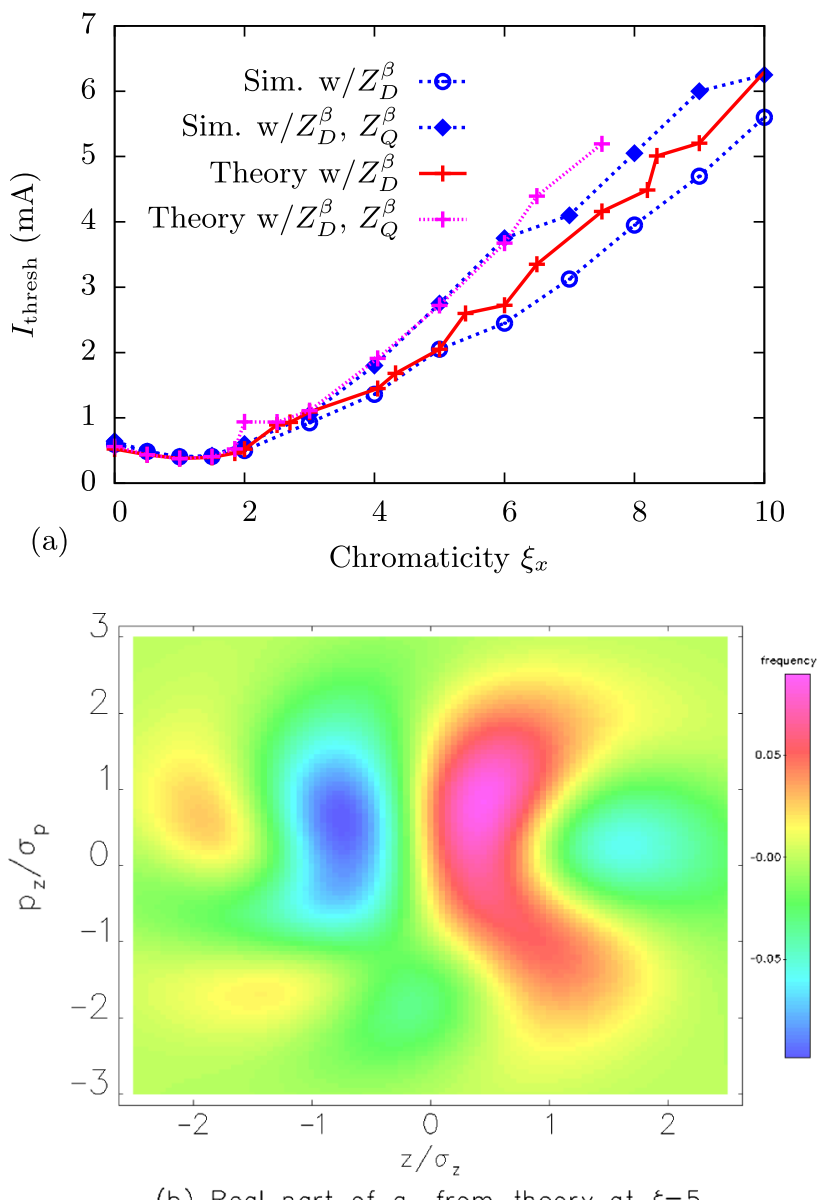

(b) Real part of $g_{1}$ from theory at $\xi=5$

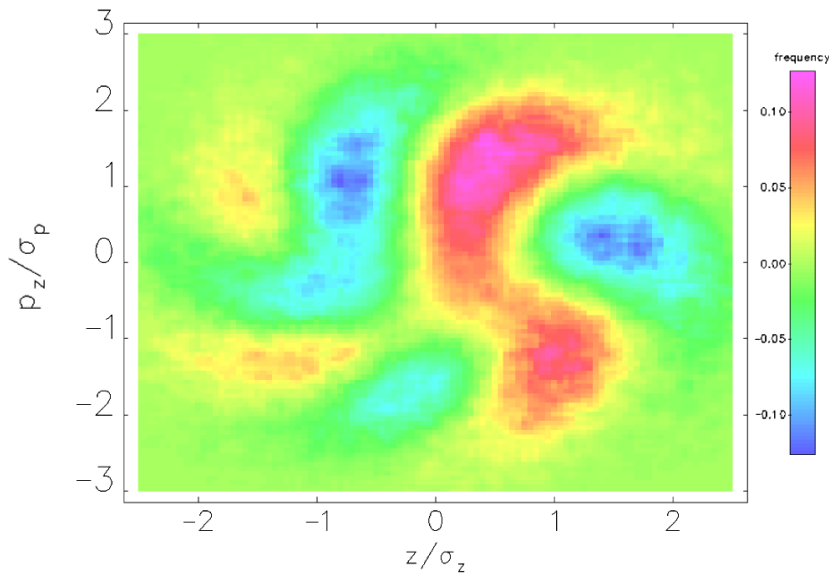

(c) Real part of $g_{1}$ from simulation at $\xi=5$

FIG. 6. Comparison of theory and simulation including the quadrupolar impedance. (a) plots the current limit with and without the quadrupolar impedance, which for the resistive wall elements of Table I has $Z_{Q}^{\beta}(k) \approx-Z_{D}^{\beta}(k) / 2$. Including $Z_{Q}$ increases $I_{\text {thresh }}$ by $10-40 \%$ at large chromaticity. (b) plots the real part of the unstable growing mode obtained from the simulation with $\xi_{x}=5$, which should be compared to the theory in panel (c). The unstable $\tilde{g}_{1}$ has $\sim 35 \%$ of the basis functions with $m=-4$ and $\sim 30 \%$ with $m=-3$, and a broader range of azimuthal variation then the purely dipolar impedance example of Fig. 4. 

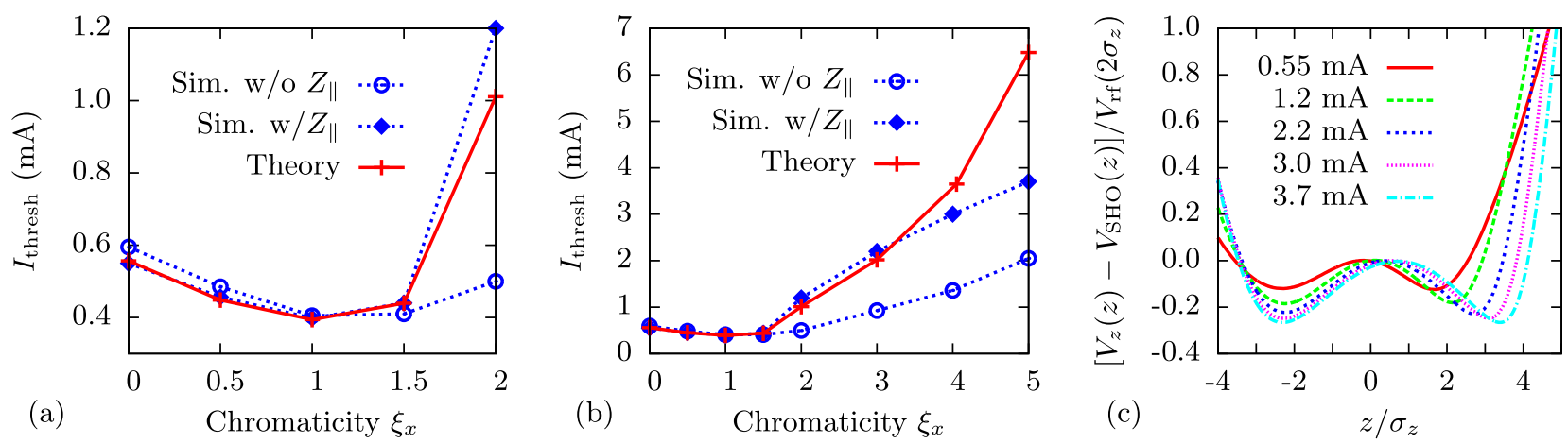

FIG. 7. Effect of adding a longitudinal impedance $Z_{\|}$. Panel (a) shows that both theory and simulation predict a reduction in $I_{\text {thresh }}$ at low $\xi_{x}$, which can be attributed to the decrease in the effective synchrotron frequency. At higher $\xi_{x}$ and $I$, (b) shows that the bunch lengthening due to $Z_{\|}$increases the maximum stable current, and the theory agrees well with the simulation for $\xi_{x} \lesssim 4$ ( $I \lesssim 3 \mathrm{~mA}$ ); for larger currents the theory overestimates $I_{\text {thresh. }}$. Panel (c) shows the difference in the longitudinal potential $V_{z}$ from that of the assumed simple harmonic oscillator at the simulation-predicted $I_{\text {thresh }}$ for $\xi_{x}=0,2,3,4$, and 5 .

$$
Z_{\|}(k)=\oint d s \frac{1-\operatorname{sgn}(k) i}{4 \pi b(s)} \sqrt{2 Z_{0} \rho(s)|k|},
$$

which is valid for both round and flat chambers.

We compare the predicted instability threshold current from theory and simulation in Fig. 7. Panel (a) shows that adding the effects of $Z_{\|}$decreases $I_{\text {thresh }}$ by about $10 \%$ at zero chromaticity. This is because $Z_{\|}$flattens the longitudinal potential, thereby decreasing the effective synchrotron frequency difference between the $m=0$ and $m=-1$ modes, so that mode coupling occurs at a lower current. Increasing the chromaticity beyond one unit stabilizes these low-order modes, and one enters the regime where the bunch lengthening provided by the longitudinal impedance increases the stability threshold $I_{\text {thresh }}$.

Figure 7(b) shows how the longitudinal impedance can significantly enhance the stability limit at higher chromaticity. This is because the nominal $I_{\text {thresh }}$ increases when $\xi_{x} \gtrsim 1.5$, and at higher current the effects of $Z_{\|}$become more pronounced. For chromaticities between 2 and 4 units the theory and simulation agree quite well, with both showing that the bunch lengthening provided by $Z_{\|}$can approximately double the maximum stable current for $2 \lesssim \xi_{x} \lesssim 4$. On the other hand, for currents above $3 \mathrm{~mA}$ $\left(\xi_{x}>4\right)$, the potential well distortion from $Z_{\|}$results in a $V_{z}$ that is not well approximated by a simple harmonic oscillator, and the theory overpredicts $I_{\text {thresh }}$. Figure 7(b) indicates that the theoretical $I_{\text {thresh }}$ is high by more than $70 \%$ at $\xi_{x}=5$, and the agreement only worsens at higher chromaticity (and current).

We quantify the change in $V_{z}$ as a function of $I$ in Fig. 7(c). Here, we plot the difference in the total ( $\mathrm{rf}+$ impedance) potential $V_{z}$ from the theoretically assumed simple harmonic oscillator $V_{\mathrm{SHO}}$, scaled by the rf potential at $z=2 \sigma_{z}$. The equilibrium potential $V_{z}$ was determined by solving the Haïssinski equation [37] with the ELEGANT-associated program HAISSINSKI written by M. Borland and L. Emery, and Fig. 7(c) plots $\Delta V_{z}$ for current values that correspond to the simulation-predicted $I_{\text {thresh }}$ for $\xi_{x}=0,2,3,4$, and 5 . At these values of $I$ the bunch length was found to be a factor of 1.12, 1.26, 1.44, 1.56, and 1.66 times the $\sigma_{z}$ at zero current, while $\sigma_{\delta}$ remained unchanged. Hence, our simulations appear to indicate that the simple harmonic approximation holds if the impedance-induced bunch-lengthening is of order $50 \%$ or less. Figure 7(c) indicates that this corresponds to a potential well distortion of order $0.2 V_{\mathrm{rf}}\left(2 \sigma_{z}\right)$, although there is no clear indication when the theory begins to make poor predictions. We expect that by more accurately describing $V_{z}$ we could make better predictions, but we do not see any other way to include $Z_{\|}$ within a tractable theory.

\section{CONCLUSIONS}

We have presented an analysis of single-bunch transverse instabilities in storage rings. Our theory is based on the linearized Fokker-Planck equation, with the solution given in terms of an eigenvalue problem for the normal modes of the beam in the presence of transverse wakefields. While the usual Vlasov analysis applies at low chromaticity, at large chromaticity many higher-order azimuthal and radial modes contribute to the dynamics, in which case including the damping and diffusion associated with synchrotron emission becomes critical to correctly predict stability. Indeed, we find that the single-bunch stability of the APS-U MBA lattice is nearly unchanged whether or not transverse nonlinearities and, hence, Landau damping, are included. Rather, the emission of synchrotron radiation supplies the dominant effective damping to the higher-order modes, and calculating this effect requires the Fokker-Planck terms. Similar conclusions will apply to any storage ring for which synchrotron emission is sufficiently strong, which will typically apply to high energy electron storage rings designed for x-ray light source applications. 


\section{ACKNOWLEDGMENTS}

We would like to thank M. Borland, L. Emery, V. Sajaev, M. Sangroula, and A. Zholents for useful discussions. This work was supported by U.S. Department of Energy Office of Sciences under Contract No. DE-AC02-06CH11357.

\section{APPENDIX A: DERIVATION OF THE FOKKER- PLANCK EQUATION AND ITS DAMPING AND DIFFUSION TERMS}

We begin with the exact, microscopic distribution function of electrons on phase space, which we denote as $\mathcal{F}(\mathcal{Z})$, where we recall that the phase space coordinates $\mathcal{Z} \equiv(\boldsymbol{X}, \boldsymbol{P})$. By "exact" and "microscopic" we mean that $\mathcal{F}(\mathcal{Z})$ tracks all the particle positions and momenta, and therefore encodes all the classical information about the electron beam. Since particle number is conserved, $\mathcal{F}$ satisfies the continuity equation

$\frac{\partial \mathcal{F}}{\partial s}+\frac{\partial}{\partial \mathcal{Z}} \cdot\left(\mathcal{Z}^{\prime} \mathcal{F}\right)=\frac{\partial \mathcal{F}}{\partial s}+\frac{\partial}{\partial \boldsymbol{X}} \cdot\left(\boldsymbol{X}^{\prime} \mathcal{F}\right)+\frac{\partial}{\partial \boldsymbol{P}} \cdot\left(\boldsymbol{P}^{\prime} \mathcal{F}\right)=0$,

where the prime indicates a derivative with respect to $s$. While exact, (A1) has far more information than we want/ could use, in that its dynamics tracks each electron trajectory. What we really are interested in is ensemble averaged quantities, which we denote here by angular brackets. We take the ensemble average of (A1) and note that $\left\langle\boldsymbol{X}^{\prime} \mathcal{F}\right\rangle=\left\langle\boldsymbol{X}^{\prime}\right\rangle\langle\mathcal{F}\rangle$ while $\left\langle\boldsymbol{P}^{\prime} \mathcal{F}\right\rangle \neq\left\langle\boldsymbol{P}^{\prime}\right\rangle\langle\mathcal{F}\rangle$ to obtain

$$
\begin{aligned}
& \frac{\partial}{\partial s}\langle\mathcal{F}\rangle+\frac{\partial}{\partial \boldsymbol{X}} \cdot\left(\left\langle\boldsymbol{X}^{\prime}\right\rangle\langle\mathcal{F}\rangle\right)+\frac{\partial}{\partial \boldsymbol{P}} \cdot\left(\left\langle\boldsymbol{P}^{\prime}\right\rangle\langle\mathcal{F}\rangle\right) \\
& =\frac{\partial}{\partial \boldsymbol{P}} \cdot\left[\left\langle\boldsymbol{P}^{\prime}\right\rangle\langle\mathcal{F}\rangle-\left\langle\boldsymbol{P}^{\prime} \mathcal{F}\right\rangle\right] .
\end{aligned}
$$

We introduce the short-hand $F \equiv\langle\mathcal{F}\rangle$ for the ensemble averaged distribution, and choose the left-hand side to contain Hamiltonian dynamics governed by external magnets, longitudinal rf focusing, and mean-field wakefields; in this case

$$
\frac{\partial}{\partial \boldsymbol{X}} \cdot\left(\left\langle\boldsymbol{X}^{\prime}\right\rangle\langle\mathcal{F}\rangle\right)+\frac{\partial}{\partial \boldsymbol{P}} \cdot\left(\left\langle\boldsymbol{P}^{\prime}\right\rangle\langle\mathcal{F}\rangle\right)=\{F, \mathcal{H}\},
$$

where $\mathcal{H}$ is the mean-field Hamiltonian determined by external forces and the ensemble averaged distribution $F$. The right-hand side of (A2) can in general contain many effects, but we will only include changes to $F$ associated with the stochastic emission of synchrotron radiation and the energy replacement delivered by the rf cavities. Therefore, we write

$$
\left.\frac{\partial}{\partial \boldsymbol{P}} \cdot\left[\left\langle\boldsymbol{P}^{\prime}\right\rangle\langle\mathcal{F}\rangle-\left\langle\boldsymbol{P}^{\prime} \mathcal{F}\right\rangle\right] \rightarrow \frac{\partial F}{\partial s}\right|_{\text {F.P. }}
$$

where the right-hand side represents a to be determined operator acting on $F$ that accounts for synchrotron radiation and the acceleration needed to replace the average energy emitted. Substituting Eqs. (A4) and (A3) into the continuity equation (A2), we find that

$$
\frac{\partial F}{\partial s}+\{F, \mathcal{H}\}=\left.\frac{\partial F}{\partial s}\right|_{\text {F.P. }}
$$

Equation (A5) governs the evolution of the ensemble averaged distribution function $F$. If we were to set the righthand side to zero, we would obtain the usual Vlasov equation in Hamiltonian form. To determine how $F$ changes due to the emission of synchrotron radiation, we will adapt the Fokker-Planck derivation presented in Ref. [38] to our storage ring. We consider an incremental step $\Delta s$ along the design orbit that has many such emission events, and describe the change in $F$ due to synchrotron emission with a probability distribution $\mathcal{P}$ that has no memory of previous emission events (technically, this models synchrotron emission as a Markov process). Thus, we write $\mathcal{P}\left(p_{z}, s ; \Delta p_{z}, \Delta s\right)$ as the probability that a particle whose negative energy deviation is $p_{z}$ at location $s$ will change its energy by an amount $\Delta p_{z}$ over the distance $\Delta s$. The distribution just after synchrotron emission is related to that just prior via

$$
\begin{aligned}
F(\mathcal{Z} ; s)= & \int d \Delta p_{z} \mathcal{P}\left(p_{z}-\Delta p_{z}, s-\Delta s ; \Delta p_{z}, \mathcal{C}_{R}\right) \\
& \times F\left(\boldsymbol{x}, z, \boldsymbol{x}^{\prime}, p_{z}-\Delta p_{z} ; s-\Delta s\right) \\
\approx & \int d \Delta p_{z}\left\{\mathcal{P} F-\Delta s \frac{\partial}{\partial s}(\mathcal{P} F)\right. \\
& \left.-\left[\Delta p_{z} \frac{\partial}{\partial p_{z}}-\frac{\left(\Delta p_{z}\right)^{2}}{2} \frac{\partial^{2}}{\partial p_{z}^{2}}\right](\mathcal{P} F)\right\},
\end{aligned}
$$

where we have Taylor expanded assuming that the energy change $\Delta p_{z}$ due to emission is small. Using the fact that the integral of the probability distribution function is unity, i.e., $\int d \Delta p_{z} \mathcal{P}=1$, we see that the first term on the right-hand side of (A6) cancels the left-hand side, while

$$
\int d \Delta p_{z} \frac{\partial}{\partial s}\left[\mathcal{P}\left(p_{z}, s ; \Delta p_{z}, \Delta s\right) F=\left.\frac{\partial F}{\partial s}\right|_{\mathrm{SR}} .\right.
$$

We reorder Eq. (A6) to find that

$$
\begin{aligned}
\left.\frac{\partial F}{\partial s}\right|_{\mathrm{SR}} \approx & -\frac{\partial}{\partial p_{z}}\left[\frac{\left\langle\Delta p_{z}\right\rangle}{\Delta s} F(\mathcal{Z} ; s)\right]_{\mathrm{SR}} \\
& +\frac{1}{2} \frac{\partial^{2}}{\partial p_{z}^{2}}\left[\frac{\left\langle\left(\Delta p_{z}\right)^{2}\right\rangle}{\Delta s} F(\mathcal{Z} ; s)\right]_{\mathrm{SR}} .
\end{aligned}
$$

Equation (A8) divides the change in $F$ into two terms: the first is damping of $p_{z}$, which physically comes about 
because higher energy electrons radiate more; the second term is a diffusive spreading in energy due to the stochastic nature of synchrotron emission.

The term associated with $\mathrm{rf}$ acceleration differs in that it has no stochastic/diffusive component. Rather, the rf serves to replenish the average longitudinal momentum lost from the emission of synchrotron radiation. Since the acceleration leaves the transverse momentum unchanged effectively decreases the electron angle, and we write

$$
\begin{aligned}
\left.\frac{\partial F}{\partial s}\right|_{\mathrm{rf}} \approx & -\frac{\partial}{\partial p_{z}}\left[\frac{\left\langle\Delta p_{z}\right\rangle}{\Delta s} F(\mathcal{Z} ; s)\right]_{\mathrm{rf}} \\
& -\frac{\partial}{\partial \boldsymbol{x}^{\prime}} \cdot\left[\frac{\left\langle\Delta \mathbf{x}^{\prime}\right\rangle}{\Delta s} F(\mathcal{Z} ; s)\right]_{\mathrm{rf}} .
\end{aligned}
$$

The first term will be chosen to cancel the mean energy lost in the ring (A8), while the second term is a source of transverse damping. The Fokker-Planck operator is the sum of (A8) and (A9) averaged over the ring,

$$
\left.\frac{\partial F}{\partial s}\right|_{\mathrm{F} . \mathrm{P} .}=\frac{1}{\mathcal{C}_{R}} \oint d s\left(\left.\frac{\partial F}{\partial s}\right|_{\mathrm{SR}}+\left.\frac{\partial F}{\partial s}\right|_{\mathrm{rf}}\right),
$$

which in turn becomes (24). To write these expressions in terms of transverse action-angle coordinates, we must transform to betatron coordinates and then action-angle coordinates, $\left(x, x^{\prime}\right) \rightarrow\left(x_{\beta}, x_{\beta}^{\prime}\right) \rightarrow(\Psi, \mathcal{J})$. Using the chain rule, this in turn leads to

$$
\begin{gathered}
\frac{\partial}{\partial p_{z}} \rightarrow \frac{\partial}{\partial p_{z}}-\frac{\eta_{x} \cos \Psi+\left(\frac{1}{2} \beta_{x}^{\prime} \eta_{x}-\eta_{x}^{\prime} \beta_{x}\right) \sin \Psi}{\sqrt{\beta_{x} / 2 \mathcal{J}}} \frac{\partial}{\partial \mathcal{J}} \\
+\frac{\eta_{x} \sin \Psi-\left(\frac{1}{2} \beta_{x}^{\prime} \eta_{x}-\eta_{x}^{\prime} \beta_{x}\right) \cos \Psi}{\sqrt{2 \mathcal{J} \beta_{x}}} \frac{\partial}{\partial \Psi} .
\end{gathered}
$$

Next, we show how to reduce the dissipative FokkerPlanck dynamics as indicated by Eq. (69). We do this by applying a number of identities involving the associated Laguerre polynomial $L_{q}^{n}$, including its differential equation

$$
\left[r \frac{d^{2}}{d r^{2}}+(n-r+1) \frac{d}{d r}+q\right] L_{q}^{n}(r)=0,
$$

its derivative

$$
\begin{aligned}
\frac{d}{d r} L_{q}^{n}(r) & =-L_{q-1}^{n+1}(r) \\
& =\frac{q}{r} L_{q}^{n}(r)-\frac{q+n}{r} L_{q-1}^{n}(r),
\end{aligned}
$$

and the recursion relations

$$
q L_{q}^{n}(r)=(n+1-r) L_{q-1}^{n+1}(r)-r L_{q-2}^{n+2}(r)
$$

$$
L_{q}^{n}(r)=L_{q}^{n+1}(r)-L_{q-1}^{n+1}(r)
$$

We begin with the derivatives of the first term in (69) that are $\propto e^{i n \Phi}$, writing

$$
\begin{aligned}
& {\left[r \frac{\partial^{2}}{\partial r^{2}}+(r+1) \frac{\partial}{\partial r}+1+\frac{4 r+1}{4 r} \frac{\partial^{2}}{\partial \Phi^{2}}\right]} \\
& \quad \times \frac{r^{n / 2} L_{q}^{n}(r) e^{i n \Phi}}{\sqrt{(q+n) ! / q !}}=\frac{r^{n / 2} e^{i n \Phi}}{\sqrt{(q+n) ! / q !}} \\
& \quad \times\left[r \frac{\partial^{2}}{\partial r^{2}}+(n-r+1) \frac{\partial}{\partial r}-\frac{n}{2}\right] L_{q}^{n} \\
& =\frac{r^{n / 2} e^{i n \Phi}}{\sqrt{(q+n) ! / q !}}\left(-q-\frac{n}{2}\right) L_{q}^{n} .
\end{aligned}
$$

The first line comes from applying chain rule while deferring derivatives of the Laguerre function, and the second line follows once we use the Laguerre differential equation (A12). Applying similar reasoning to the second term in Eq. (69) $\propto e^{i(n+2) \Phi}$ gives

$$
\begin{gathered}
\frac{e^{2 i \Phi}}{2}\left[r \frac{\partial^{2}}{\partial r^{2}}+r \frac{\partial}{\partial r}+i \frac{\partial^{2}}{\partial r \partial \Phi}+\frac{1-r}{2 i} \frac{\partial}{\partial \Phi}-\frac{1}{4 r} \frac{\partial^{2}}{\partial \Phi^{2}}\right] \\
\times \frac{r^{n / 2} L_{q}^{n}(r) e^{i n \Phi}}{\sqrt{(q+n) ! / q !}}=\frac{r^{n / 2} e^{i(n+2) \Phi}}{2 \sqrt{(q+n) ! / q !}}\left(r \frac{\partial^{2}}{\partial r^{2}}-r \frac{\partial}{\partial r}\right) L_{q}^{n} \\
\quad=\frac{r^{n / 2} e^{i(n+2) \Phi}}{2 \sqrt{(q+n) ! / q !}}\left[-(n+1) \frac{\partial}{\partial r}-q\right] L_{q}^{n},
\end{gathered}
$$

while the third term $\propto e^{i(n-2) \Phi}$ reduces to

$$
\begin{aligned}
& \frac{e^{-2 i \Phi}}{2}\left[r \frac{\partial^{2}}{\partial r^{2}}+r \frac{\partial}{\partial r}-i \frac{\partial^{2}}{\partial r \partial \Phi}-\frac{1-r}{2 i} \frac{\partial}{\partial \Phi}-\frac{1}{4 r} \frac{\partial^{2}}{\partial \Phi^{2}}\right] \\
& \quad \times \frac{r^{n / 2} L_{q}^{n}(r) e^{i n \Phi}}{\sqrt{(q+n) ! / q !}} \\
& =\frac{r^{n / 2} e^{i(n-2) \Phi}}{2 \sqrt{(q+n) ! / q !}}\left[r \frac{\partial^{2}}{\partial r^{2}}+(2 n-r) \frac{\partial}{\partial r}-n+\frac{n(n-1)}{r}\right] L_{q}^{n}
\end{aligned}
$$

$$
=\frac{r^{n / 2} e^{i(n-2) \Phi}}{2 \sqrt{(q+n) ! / q !}}\left[(n-1) \frac{\partial}{\partial r}-(q+n)+\frac{n(n-1)}{r}\right] L_{q}^{n} .
$$

The diagonal term Eq. (A18) is precisely the one listed in (69), which gives rise to the damping $(2 p+m) / \tau_{z}$ of the linear mode $(p, m)$. The second contribution can be simplified by first applying the derivative equation (A13) and then the recursion relation (A15): 


$$
\begin{aligned}
& -\left[(n+1) \frac{\partial}{\partial r}+q\right] L_{q}^{n}=(n+1) L_{q-1}^{n+1} \\
& -(n+1-r) L_{q-1}^{n+1}+r L_{q-2}^{n+2} \\
& =r\left(L_{q-1}^{n+1}+L_{q-2}^{n+2}\right) .
\end{aligned}
$$

Finally, applying the recursion relation (A16) to (A20) implies that

$$
\begin{gathered}
\frac{r^{n / 2} e^{i(n+2) \Phi}}{2 \sqrt{(q+n) ! / q !}}\left[-(n+1) \frac{\partial}{\partial r}-q\right] L_{q}^{n} \\
=\frac{r^{n / 2} e^{i(n+2) \Phi}}{2 \sqrt{(q+n) ! / q !}} r L_{q-1}^{n+2}(r)
\end{gathered}
$$

as we wrote in (69). We deal with (A22) by first using the derivative (A14) to write

$$
\begin{aligned}
& {\left[(n-1) \frac{\partial}{\partial r}-(q+n)+\frac{n(n-1)}{r}\right] L_{q}^{n}} \\
& \quad=\frac{(n-1)(q+n)}{r}\left(L_{q}^{n}-L_{q-1}^{n}\right)-(n+q) L_{q}^{n} \\
& \quad=\frac{(n-1)(q+n)}{r} L_{q}^{n-1} \\
& -\frac{n+q}{r}\left[(q+1) L_{q+1}^{n-1}-(q+n) L_{q}^{n-1}\right] \\
& =\frac{(q+n)(q+1)}{r}\left(L_{q}^{n-1}-L_{q+1}^{n-1}\right)
\end{aligned}
$$

where (A26) follows from equating the differential relations (A13) and (A14) and using the recursion relation (A16), while the final line collects terms. Now, we make one final application of Eq. (A16) to conclude that

$$
\begin{aligned}
& \frac{r^{n / 2} e^{i(n-2) \Phi}}{2 \sqrt{(q+n) ! / q !}}\left[(n-1) \frac{\partial}{\partial r}-(q+n)+\frac{n(n-1)}{r}\right] L_{q}^{n} \\
& =\frac{r^{n / 2} e^{i(n-2) \Phi}}{2 \sqrt{(q+n) ! / q !}} \frac{(q+n)(q+1)}{r} L_{q+1}^{n-2}
\end{aligned}
$$

\section{APPENDIX B: BETATRON OSCILLATIONS OF A KICKED BEAM INCLUDING DAMPING AND DIFFUSION}

In this Appendix we consider the evolution of a kicked beam in the limit of no wakefields, $\chi \rightarrow 0$. Ultimately we will be interested in the betatron oscillation of the beam, so that we will need to differentiate between the physically measurable betatron phase $\Psi$ and its head-tail (chromatic) counterpart $\bar{\Psi}=\Psi+k_{\xi} z$. In particular, the initial distribution of the kicked beam is

$$
\begin{aligned}
F_{1}(s=0)= & -\mathcal{D}(0) \sqrt{\frac{1}{2}} \mathcal{J} f_{0}^{\prime}(\mathcal{J}) e^{i \Psi} \frac{e^{-r}}{2 \pi} \\
= & -\sqrt{\frac{1}{2} \mathcal{J} f_{0}^{\prime}(\mathcal{J}) e^{i \Psi}} \\
& \times \mathcal{D}(0) \sum_{\ell} i^{-\ell} J_{\ell}\left(k_{\xi} \sigma_{z} \sqrt{2 r}\right) e^{i \ell \Phi} \frac{e^{-r}}{2 \pi}
\end{aligned}
$$

The first line of (B1) describes the transverse dependence in terms of the head-tail shifted betatron phase, while the second line contains the initial value of the longitudinal distribution function. The sum over angular harmonics shows that the initial mode content depends on the chromaticity, because the linear modes are defined with respect to the head-tail (chromatic) shifted betatron frequency.

We calculate the mode coefficients by setting the second line in (B1) equal to the perturbation $\tilde{g}_{1}$ :

$$
\begin{aligned}
\tilde{g}_{1}(\Phi, r) & =\sum_{q, n} a_{q}^{n} \frac{r^{n / 2} L_{q}^{n}(r) e^{-r}}{\sqrt{(q+n) ! / q !}} \frac{e^{i n \Phi}}{2 \pi} \\
& =\mathcal{D}(0) \sum_{\ell} i^{-\ell} J_{\ell}\left(k_{\xi} \sigma_{z} \sqrt{2 r}\right) e^{i \ell \Phi} \frac{e^{-r}}{2 \pi} .
\end{aligned}
$$

We isolate the mode coefficients by multiplying both sides by $r^{m / 2} L_{p}^{m}(r) e^{-r} e^{-i m \Phi}$ and integrating over $\Phi$ and $r$. Using the Laguerre orthogonality relation (71) and our favorite Laguerre-Bessel integral (61), we find that

$$
a_{p}^{m}=\mathcal{D}(0) \frac{\left(k_{\xi} \sigma_{z} / \sqrt{2}\right)^{2 p+m}}{i^{m} \sqrt{p !(p+m) !}} e^{-k_{\xi}^{2} \sigma_{z}^{2} / 2}
$$

The evolution of the kicked beam is therefore given by

$$
\begin{aligned}
F_{1}= & -\sqrt{\frac{1}{2} \mathcal{J}} f_{0}^{\prime}(\mathcal{J}) e^{i \bar{\Psi}} e^{-i \omega_{\beta} s / c} \\
& \times \sum_{p, m} a_{p}^{m} e^{-i \Omega s / c} \frac{r^{m / 2} L_{p}^{m}(r) e^{-r}}{\sqrt{(p+m) ! / p !}} \frac{e^{i m \Phi}}{2 \pi}
\end{aligned}
$$

with $a_{p}^{m}$ listed in Eq. (B4).

To find how the dipole moment associated with the transverse betatron oscillation evolves, we multiply (B5) by $\sqrt{2 \mathcal{J}} e^{-i \Psi} e^{i \omega_{\beta} s / c}$ and integrate over all phase space. Note that here we use the physically measurable betatron phase rather than its head-tail shifted counterpart. Applying a similar calculation to that which led to (B4) gives us the dipole moment 


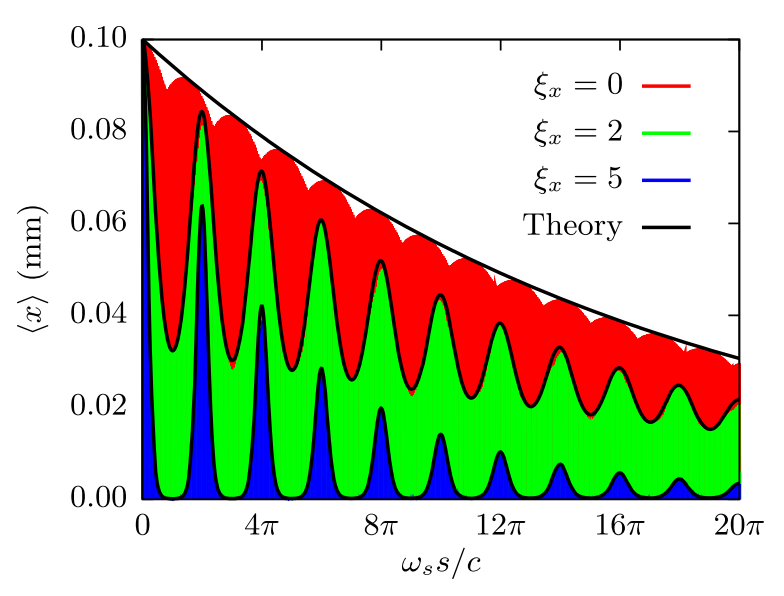

FIG. 8. Evolution of the dipole moment $\langle x\rangle$ for three different chromaticities when one includes Fokker-Planck damping and diffusion. The colored lines indicate simulation results using the parameters from Fig. 1, which has $\omega_{s} \tau_{x} \approx 53.1, \omega_{s} \tau_{z} \approx 61.9$, and $k_{\xi} \sigma_{z} / \xi_{x} \approx 0.37$. For this lattice, the nonlinear tune shift with amplitude is negligible at these small oscillation amplitudes.

$$
\begin{aligned}
\mathcal{D}(s) & =\int d \mathcal{Z} \sqrt{2 \mathcal{J}} e^{-i\left(\Psi-k_{\xi} z\right)} F_{1} e^{i \omega_{\beta} s / c} \\
& =\sum_{p, m} a_{p}^{m} e^{-i \Omega s / c} \frac{i^{m}\left(k_{\xi} \sigma_{z} / \sqrt{2}\right)^{2 p+m}}{\sqrt{p !(p+m) !}} e^{-k_{\xi}^{2} \sigma_{z}^{2} / 2} \\
& =\mathcal{D}(0) \sum_{p, m} e^{-i \Omega s / c} \frac{\left(k_{\xi}^{2} \sigma_{z}^{2} / 2\right)^{2 p+m}}{p !(p+m) !} e^{-k_{\xi}^{2} \sigma_{z}^{2}}
\end{aligned}
$$

Now, we use the fact that the eigenvalue $\Omega$ is approximately given by the diagonal contribution

$$
\Omega \approx m \omega_{s}-\frac{i}{\tau_{x}}-\frac{i}{\tau_{z}}(2 p+m),
$$

and take care regarding the summation limits to write

$$
\begin{aligned}
\mathcal{D}(s)= & \mathcal{D}(0) e^{-s / c \tau_{x}} e^{-k_{\xi}^{2} \sigma_{z}^{2}} \\
& \times \sum_{p=0}^{\infty} \sum_{m=-p}^{\infty} \frac{e^{-i m \omega_{s} s / c}}{p !(p+m) !}\left(\frac{k_{\xi}^{2} \sigma_{z}^{2}}{2} e^{-s / \tau_{z} c}\right)^{2 p+m} .
\end{aligned}
$$

By changing the summation variable from $m$ to $n=m+p$ we end up having the product of two separate sums that have the form $\sum_{n=0}^{\infty} a^{n} / n !=e^{a}$. Hence, the dipole oscillation

$$
\begin{aligned}
\mathcal{D}(s)= & \mathcal{D}(0) e^{-s / c \tau_{x}} \\
& \times \exp \left\{-k_{\xi}^{2} \sigma_{z}^{2}\left[1-e^{-s / c \tau_{z}} \cos \left(\omega_{s} s / c\right)\right]\right\} .
\end{aligned}
$$

In the limit of no synchrotron damping, $\tau_{z} \rightarrow 0$, the formula (B10) equals $e^{-s / c \tau_{x}}$ times the kicked beam expression derived in Ref. [39]. In addition to the slow transverse damping, the measured betatron oscillation displays amplitude modulations at the synchrotron frequency. This modulation arises because the accumulated betatron phase depends on the longitudinal position through the head-tail effect, while its depth scales with the chromaticity.

When synchrotron damping is included, Eq. (B10) shows that the time over which the synchrotron amplitude modulations occur decreases with chromaticity, which in turn effectively damps the coherent betatron motion. This apparent damping arises from the fact that the mode content of an initially kicked beam depends on $\xi_{x}$ : the number of higher-order modes increases with chromaticity, and higher-order modes are more heavily damped. Note that in a real machine it may be difficult to untangle the effect described by Eq. (B10) from a similar damping-like decoherence that is due to the nonlinear tune-shift with amplitude [39]; the differences should appear at small betatron oscillation amplitudes that may be difficult to accurately prepare and measure. In simulation, however, it is quite easy to detect the difference. We show an example using APS-U parameters in Fig. 8.

[1] R. D. Kohaupt, Single beam instabilities in PETRA, IEEE Trans. Nucl. Sci. 26, 3480 (1979).

[2] J. M. Byrd, R. Holtzapple, and B. Podobedov, Singlebunch collective effects in the advanced light source, AIP Conf. Proc. 367, 236 (1996).

[3] K. C. Harkay, M. Borland, Y.-C. Chae, L. Emery, Z. Huang, E. S. Lessner, A. H. Lumpkin, S. V. Milton, N.S. Sereno, and B.X. Yang, in Proceedings of the 18th Particle Accelerator Conference, New York, 1999 (IEEE, New York, 1999), p. 1644.

[4] R. Nagaoka, J. L. Revol, P. Kernel, and G. Besnier, in Proceedings of the 18th Particle Accelerator Conference, New York, 1999 (IEEE, New York, 1999), p. 1192.

[5] F. Sacherer, Report No. 72-5, CERN/SI-BR, 1972.

[6] F. Sacherer, Report No. 76-21, CERN/PS-BR, 1976.

[7] A. W. Chao, Physics of Collective Beam Instabilities in High Energy Accelerators (Wiley, New York, 1993).

[8] K. Y. Ng, Physics of Intensity Dependent Beam Instabilities (World Scientific, Singapore, 2006).

[9] A. Burov, Nested head-tail vlasov solver, Phys. Rev. ST Accel. Beams 17, 021007 (2014).

[10] T. Suzuki, Fokker-Planck theory of transverse modecoupling instability, Part. Accel. 20, 79 (1986).

[11] M. Sands, SLAC Report No. 121, 1970.

[12] T. Suzuki, Hamiltonian formulation for synchrotron oscillations and Sacherer's integral equation, Part. Accel. 12, 237 (1982).

[13] J. S. Berg, Ph.D. thesis, Stanford University, 1996; Report No. SLAC-R-478.

[14] N. Mounet, Ph.D. thesis, École Polytechnique Fédérale de Lausanne, 2012. 
[15] M. Borland, Advanced Light Source Technical Report No. LS-287, 2000.

[16] M. Borland, V. Sajaev, and Y. Sun, in Proc. of the 2015 IPAC, Richmond, VA, 2015 (JACoW, Geneva Switzerland, 2015), p. 1776, TUPJE063.

[17] R. D. Kohaupt, DESY Report No. 80/22, 1980.

[18] B. Zotter, CERN/ISR-TH Report No. 82-10, 1982.

[19] A. W. Chao, SLAC Report No. 2946, 1982.

[20] K. Satoh and Y. Chin, Transverse mode coupling in a bunched beam, Nucl. Instrum. Methods Phys. Res., Sect. A 207, 309 (1983).

[21] P. Kernel, R. Nagaoka, J.-L. Revol, and G. Besnier, in Proceedings of the European Particle Accelerator Conference, Vienna, 2000 (EPS, Geneva, 2000), p. 1133.

[22] A. Burov and V. Danilov, Suppression of Transverse Bunch Instabilities by Asymmetries in the Chamber Geometry, Phys. Rev. Lett. 82, 2286 (1999).

[23] H. Wiedemann, Particle Accelerator Physics II, 2nd ed. (Springer-Verlag, Berlin, 1999).

[24] E. D. Courant and H. S. Snyder, Theory of the alternatinggradient synchrotron, Ann. Phys. (N.Y.) 3, 1 (1958).

[25] H. Wiedemann, Particle Accelerator Physics I (SpringerVerlag, Berlin, 1993).

[26] S. Heifets, A. Wagner, and B. Zotter, SLAC Report No. AP110, 1998.

[27] H. Goldstein, Classical Mechanics, 2nd ed. (AddisonWesley, Reading, MA, 1980).

[28] I. Percival and D. Richards, Introduction to Dynamics (Cambridge University Press, Cambridge, England, 1982).
[29] C. Pellegrini, On a new instability in electron-positron storage rings (the head-tail effect), Nuovo Cimento 64, 447 (1969).

[30] M. Sands, SLAC Report No. 8, SLAC, 1969.

[31] B. Nash, Ph.D. thesis, Stanford University, 2006; Report No. SLAC-R-820.

[32] I. S. Gradshteyn and I. M. Ryzhik, Table of Integrals, Series, and Products, 6th ed. (Academic Press, New York, 2000).

[33] Y. Wang and M. Borland, Pelegant: A parallel accelerator simulation code for electron generation and tracking, AIP Conf. Proc. 877, 241 (2007).

[34] L. J. Laslett, V. K. Neil, and A. M. Sessler, Transverse resistive instabilities of intense coasting beams in particle accelerators, Rev. Sci. Instrum. 36, 436 (1965).

[35] R. L. Gluckstern, J. van Zeits, and B. Zotter, Coupling impedance of beam pipes of general cross section, Phys. Rev. E 47, 656 (1993).

[36] J. L. Laclare, CERN Accelerator School: Advanced Accelerator Physics (CERN, Geneva, Switzerland, 1985), p. 264.

[37] J. Haïssinski, Exact longitudinal equilibrium distribution of stored electrons in the presence of self-fields, Nuovo Cimento Soc. Ital. Fis. 18, 72 (1973).

[38] R. D. Hazeltine and F. L. Waelbroeck, The Framework Of Plasma Physics, 1st ed. (Westview Press, Boulder, CO, 1998).

[39] R. E. Meller, A. W. Chao, J. M. Peterson, S. G. Peggs, and M. Furman, SSC Report No. N-360, 1987. 